\title{
Effect of Stimulus-Dependent Spike Timing on Population Coding of Sound Location in the Owl's Auditory Midbrain
}

\author{
기. V. Beckert, ${ }^{1}$ B B. J. Fischer, ${ }^{2}$ and J. L. Pena ${ }^{1}$ \\ https://doi.org/10.1523/ENEURO.0244-19.2020 \\ ${ }^{1}$ Dominick P. Purpura Department of Neuroscience, Albert Einstein College of Medicine, Bronx, NY 10461 and \\ ${ }^{2}$ Department of Mathematics, Seattle University, Seattle, WA 98122
}

\begin{abstract}
In the auditory system, the spectrotemporal structure of acoustic signals determines the temporal pattern of spikes. Here, we investigated this effect in neurons of the barn owl's auditory midbrain (Tyto furcata) that are selective for auditory space and whether it can influence the coding of sound direction. We found that in the nucleus where neurons first become selective to combinations of sound localization cues, reproducibility of spike trains across repeated trials of identical sounds, a metric of across-trial temporal fidelity of spiking patterns evoked by a stimulus, was maximal at the sound direction that elicited the highest firing rate. We then tested the hypothesis that this stimulus-dependent patterning resulted in rate co-modulation of cells with similar frequency and spatial selectivity, driving stimulus-dependent synchrony of population responses. Tetrodes were used to simultaneously record multiple nearby units in the optic tectum (OT), where auditory space is topographically represented. While spiking of neurons in OT showed lower reproducibility across trials compared with upstream nuclei, spike-time synchrony between nearby OT neurons was highest for sounds at their preferred direction. A model of the midbrain circuit explained the relationship between stimulus-dependent reproducibility and synchrony, and demonstrated that this effect can improve the decoding of sound location from the OT output. Thus, stimulus-dependent spiking patterns in the auditory midbrain can have an effect on spatial coding. This study reports a functional connection between spike patterning elicited by spectrotemporal features of a sound and the coding of its location.
\end{abstract}

Key words: barn owl; population coding; sound localization; spectrotemporal; synchrony

\section{Significance Statement}

Spike timing of auditory neurons is modulated by the spectrotemporal structure of sounds. Whether this effect may have implications for coding of sound location remains unresolved. This question was approached in neurons of the owl's midbrain, where temporal spiking patterns are modulated by the sound's envelope while firing rate is driven by sound direction. We found that temporal patterns were dependent on binaural cues, leading to stimulus-dependent synchrony of nearby cells. Theoretical analysis showed that stimulus-dependent temporal patterning predicts stimulus-dependent synchrony in nearby cells sharing input with similar spectrotemporal structure, which in turn can sharpen the downstream readout of sound direction. This work shows how stimulus-dependent spike timing can affect the downstream coding of sound location by firing rate, a mechanism that can be generalized to sensory neurons sensitive to the temporal structure of the stimulus.

Received June 24, 2019; accepted February 18, 2020; First published March 13, 2020.

The authors declare no competing financial interests.
Author contributions: M.V.B. and J.L.P. designed research; M.V.B. performed research; M.V.B. and B.J.F. analyzed data; M.V.B., B.J.F., and J.L.P. wrote the paper. 


\section{Introduction}

The temporal pattern of spiking of neurons in the auditory system is determined by the phase and amplitude modulations of frequency components of sound, known as "fine-structure" and "envelope," respectively (Louage et al., 2004). The temporal precision of patterned responses varies along the auditory pathway, as downstream neurons display reduced capacity of locking to the phase of the stimulus fine structure (Johnson, 1980; Winter and Palmer, 1990; Köppl, 1997; Louage et al., 2004; Liu et al., 2006) and envelope (Lu et al., 2001; Joris et al., 2004; Christianson and Peña, 2006, 2007; Steinberg and Peña, 2011). These changes can result from the increased dimensionality of the neurons' spectrotemporal tuning (Woolley et al., 2009; Kim and Doupe, 2011; Atencio et al., 2012) and emergent selectivities, such as to sound direction (Yao et al., 2015a,b).

Like other animals, owls use disparities in arrival time (interaural time difference; ITD) and level (interaural level difference; ILD) of acoustic signals between the ears to localize sound (Knudsen et al., 1979; Moiseff, 1989). ITD and ILD are processed in parallel brainstem pathways (Takahashi et al., 1984) that converge in the lateral shell of the central nucleus of the inferior colliculus (ICcl), where neurons become tuned to both ITD and ILD (Takahashi et al., 1989; Adolphs, 1993; Fig. 1). Downstream from ICcl, a topographic representation of auditory space (referred to as space-map) emerges in the external nucleus of the inferior colliculus (ICx) and the optic tectum (OT; Knudsen and Konishi, 1978a,b; Knudsen, 1982). ICx neurons receive convergent input from ICcl (Wagner et al., 1987), which sharpens their spatial tuning through frequency convergence (Takahashi and Konishi, 1986; Mazer, 1998; Peña and Konishi, 2000).

As in other species, temporal spiking patterns in the owl's auditory brainstem pathways and midbrain are determined by the sound's envelope (Keller and Takahashi, 2000; Steinberg and Peña, 2011; Steinberg et al., 2013). This patterning may facilitate the distinction between echoes and separate sources (Keller and Takahashi, 2000, 2005; Baxter et al., 2013). However, the bilateral matching of selectivity to spectrotemporal features of sounds in the inputs to ITD detector neurons (Fischer et al., 2011) and the dependence of selectivity to spectrotemporal features on ILD (Steinberg et al., 2013) in lower stages of the pathway suggests that this selectivity may depend on binaural

This work was supported by the National Institutes of Health Grant DC007690.

Acknowledgements: We thank Adam Kohn and Ruben Coen-Cagli for feedback on the analysis of synchrony, Romain Brette for providing code for shuffled autocorrelograms, Gervasio Batista and Rodrigo Pavão for comments on this manuscript, and anonymous reviewers for their thoughtful and constructive feedback.

Correspondence should be addressed to J. L. Pena at jose.pena@ einsteinmed.org.

https://doi.org/10.1523/ENEURO.0244-19.2020

Copyright @ 2020 Beckert et al.

This is an open-access article distributed under the terms of the Creative Commons Attribution 4.0 International license, which permits unrestricted use, distribution and reproduction in any medium provided that the original work is properly attributed. cues. Whether this effect is conveyed to the $\mathrm{ICcl}$ is unknown.

Here, we built on previous work by investigating whether temporal spiking patterns may influence the coding of auditory space by midbrain neurons. We hypothesized that spike patterning induced by the sound's envelope is affected by ITD and ILD, thus suggesting a functional relationship between selectivity to stimulus features linked to sound identity and location. We addressed this question by examining across-trial reproducibility of spike trains in ICcl elicited by identical copies of a sound, using standard metrics of spike-train distance (Victor and Purpura, 1996; Joris et al., 2006; Brette, 2012) as ITD, ILD, and sound direction in free field were varied. Reproducibility in ICcl was correlated with firing rate, as ITD and ILD delivered through earphones and sound direction in free field were independently manipulated, reaching a maximum at the preferred stimulus parameter in all cases.

Importantly, both ICx and OT display direction-dependent frequency selectivity (Knudsen, 1984; Cazettes et al., 2014; Fig. 1). Because the sound envelope is determined by the frequency spectrum (Louage et al., 2004), similar frequency selectivity in neurons with the same spatial tuning suggests these neurons may receive inputs with a similar envelope. In addition, it has recently been shown that nearby neurons in OT may share inputs (Beckert et al., 2017). However, neurons downstream the auditory pathway typically show reduced envelope locking (Lu et al., 2001; Joris et al., 2004; Louage et al., 2004; Christianson and Peña, 2007; Steinberg and Peña, 2011; Steinberg et al., 2013; Yao et al., 2015a,b). Thus, whether temporal spiking patterns in ICcl have an effect on downstream neurons is an open question. We tested the hypothesis that while single OT neurons would display reduced ability to lock to the envelope, an effect on temporal correlations remained at the population level. To test this, tetrodes were used to simultaneously record multiple single units in OT.

Consistent with our hypothesis, synchrony of neighboring OT neurons varied in a stimulus-dependent manner. Modeling explained the relationship between stimulus-dependent spiking patterns determined by the sound's envelope and downstream synchrony, and that stimulus-dependent synchrony could affect the decoding of the OT population through disambiguating population responses. These results demonstrate an interaction between spike timing driven by spectrotemporal properties of sounds and coding of sound location that may have a role in behavioral performance.

\section{Materials and Methods}

\section{Subjects and surgery}

Adult American barn owls (Tyto furcata) of both sexes (four male, one female) were implanted with custom-built stainless steel headplates held in place with dental acrylic. A well was molded in the acrylic posterior of the headplate, aimed over ICcl and OT.

Owls were food-deprived at least $12 \mathrm{~h}$ before each procedure. Anesthesia was induced by intramuscular 


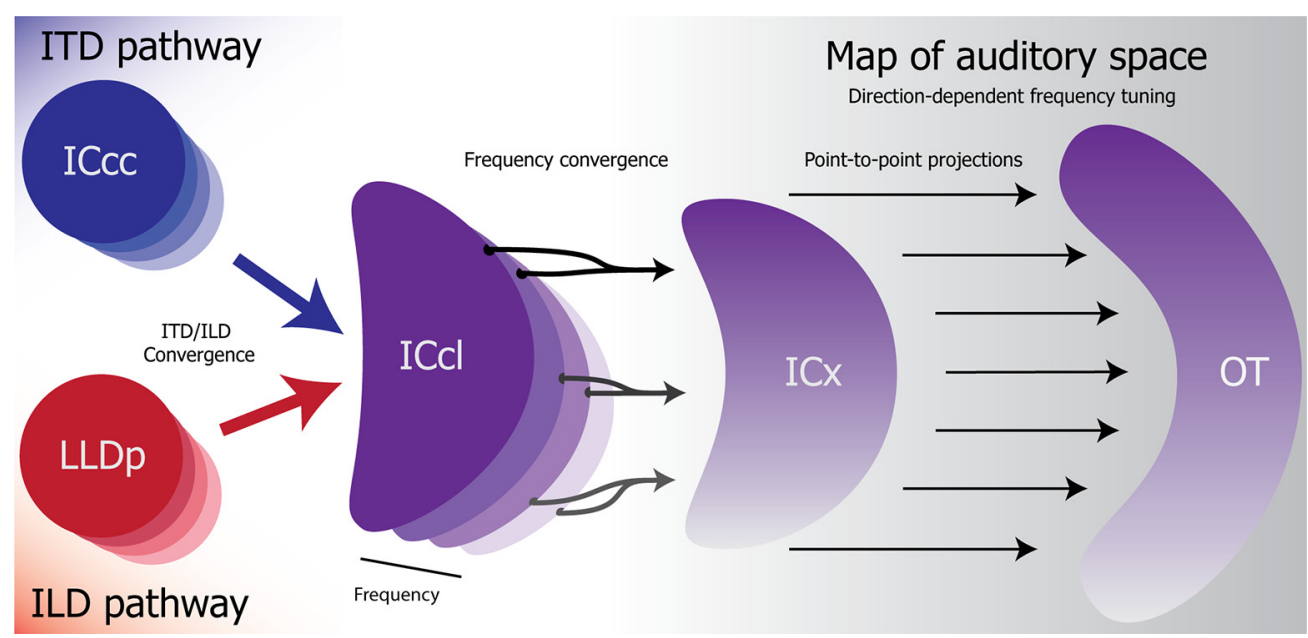

Figure 1. Schematic of the barn owl auditory midbrain. ITD (blue) and ILD (red) are computed along parallel brainstem pathways ending, respectively, in the core of the central nucleus of the inferior colliculus (ICCC) and the posterior part of the dorsal lateral lemniscus (LLDp). These regions are tonotopically organized (illustrated by contrast levels of color). The ITD and ILD pathways converge onto the lateral shell of the core of the inferior colliculus (ICcl). ICcl neurons display both ITD and ILD tuning (purple) and are also tonotopically organized. Frequency convergence onto the external nucleus of the inferior colliculus (ICx) contributes to the emergence of a map of auditory space. ICx sends point-to-point projections to the OT (Knudsen and Knudsen, 1983). Both ICx and OT display direction-dependent frequency tuning (illustrated with a purple gradient; Knudsen, 1984; Cazettes et al., 2014).

injections of ketamine (Ketaset; $20 \mathrm{mg} / \mathrm{kg}$ ) and xylazine (Anased; $2 \mathrm{mg} / \mathrm{kg}$ ). Owls were provided with prophylactic antibiotic (Ampicillin; $20 \mathrm{mg} / \mathrm{kg}$, i.m.) and subcutaneous hydration (lactated Ringer's solution; $10 \mathrm{ml}$, s.c.). A proper anesthesia level was assessed via pedal and eyelid reflex. This level of anesthesia was maintained with subsequent half-doses of ketamine and xylazine throughout the recording session. Owls were wrapped with a heating pad to maintain body temperature.

At the end of the recording session, the craniotomy was covered with a sterile plastic disk and the well was filled with a quick-dry silicone compound (Quick-Pro, Warner Tech-Care). An analgesic was administered to prevent pain after recovery from anesthesia (Rimadyl; $3 \mathrm{mg} / \mathrm{kg}$, i. $\mathrm{m}$.). Owls were allowed to recover in a small crate overnight. Once all signs of physical impairment were absent, they were returned to their home aviary. Subsequent recording sessions were always 10 or more days later to allow for proper recovery. All procedures were in compliance with guidelines set by the National Institute of Health and the Albert Einstein College of Medicine animal care committee's regulations.

\section{Data collection}

All recordings were performed in a double wall sound attenuating chamber (Industrial Acoustics) lined with anechoic acoustic foam (Sonex). ICcl and OT were targeted using known stereotaxic coordinates relative to the intersection of the midline and interaural line. Small openings in the dura were made with a sterile needle to facilitate electrode entry into the brain. Tungsten electrodes (5-M $\Omega$ resistance; A-M Systems) were driven with a microdrive (Newport) into ICcl. These high-impedance electrodes were able to isolate single units from the strong evoked field potentials of ICcl. Data acquisition was performed using a TDT system 3 (Tucker-Davis Technologies) and custom-written MATLAB routines (Tytology). A threshold was chosen for single cell isolation based on online visual monitoring of spike amplitudes.

Recordings in OT were performed using tetrodes $(\mathrm{Q}-$ trodes; NeuroNexus) driven with a Kopf microdrive (David Kopf Instruments). A Plexon Omniplex system (SortClient, Plexon) was used for data acquisition. Recordings were performed on sites containing at least two visually wellisolated units. Offline sorting software was used to confirm isolation (Offline Sorter, Plexon). On average, four to five units were recorded per site.

Electrode locations within both brain regions were confirmed using established physiological characteristics including tuning to ITD, ILD, and frequency in both ICCl (Takahashi and Konishi, 1986; Wagner et al., 1987; Takahashi et al., 1989; Bremen et al., 2007) and OT, as well as bursting discharge and response to light stimulation in OT (Knudsen, 1982, 1984).

Because of spike-sorting constraints, bursting responses, characteristic of superficial layers of OT, were excluded from the analysis. Therefore the OT units included in the analysis are likely from deep OT layers (Knudsen, 1982).

\section{Acoustic stimulation}

\section{Dichotic (earphone) stimulation}

Acoustic stimulation was performed using previously described methods. In brief, TDT system three and custom MATLAB routines were used to synthesize and deliver all stimuli. Custom-made earphones containing a speaker (Knowles, model 1914) and a microphone (Knowles, model 1319) were inserted into the owl's ear canal. Earphone speakers were calibrated using the earphone microphones each time they were positioned in the ear canals. Broadband 
sounds $(0.5-10 \mathrm{kHz}$; duration, $150 \mathrm{~ms}$; interstimulus interval, $300 \mathrm{~ms}$ ) were presented randomly for either varying ITDs or ILDs to measure tuning functions. For ITD and ILD tuning characterization, broadband stimuli were not identical in each presentation (unfrozen noise). After ITD and ILD tuning was characterized, identical broadband stimuli (frozen noise) with a longer duration $(450 \mathrm{~ms})$ presented $100-300$ times were used to assess spiking reproducibility (see Data Analysis section below). A new frozen noise token was generated for each neuron.

\section{Free-field stimulation}

Free-field sound stimulation was presented through a custom built semi-spherical array of speakers (Sennheiser, 3P127A) surrounding the stereotax. Speaker positions ranged from $\pm 100^{\circ}$ azimuth and $\pm 80^{\circ}$ elevation with spacing between $10^{\circ}$ and $30^{\circ}$. Owls were positioned to face the $0^{\circ}$ azimuth and $0^{\circ}$ elevation speaker for all recordings. Speakers were calibrated using a Brüel and Kjær microphone (model 4190). Broadband signals $(0.5-10 \mathrm{kHz})$ were transformed by the calibration filter for each speaker to equalize sounds across the array. Stimulus duration $(150 \mathrm{~ms})$ and interstimulus intervals $(300 \mathrm{~ms})$ were the same as those for dichotic stimulation. Speakers were activated randomly 20-40 times to measure a spatial receptive field (SpRF). Broadband noise stimuli used for SpRF characterization were not identical in each presentation (unfrozen noise). As for experiments using dichotic stimulation, after characterization of the SpRF, longer stimuli $(250 \mathrm{~ms})$ presented 100-300 times were used to assess reproducibility (see Data Analysis section below). A new frozen noise token was generated for each set of simultaneously recorded neurons.

\section{Data Analysis \\ Tuning curves}

ITD, ILD, and spatial tuning curves were computed from the average number of spikes elicited during sound presentation over 10-30 repetitions of broadband noise. Neurons were classified as having a significant response to auditory stimulation if the average response to any stimulus condition was 2 SD larger than the mean spontaneous firing rate, which was measured by counting spikes preceding the stimulus, during an equivalent amount of time as the stimulus duration (all neurons fit this criterion).

\section{Response reproducibility}

Across-trial reproducibility of spike trains was calculated using a shuffled autocorrelogram (SAC) as previously reported (Joris et al., 2006; Brette, 2012). Neurons in ICcl and OT characteristically display a strong onset response (Knudsen, 1984). In order to ensure measurements of reproducibility were not biased by onset responses, the first $50 \mathrm{~ms}$ after stimulus onset were excluded. SACs are histograms of time intervals between each spike in a stimulus trial and every spike in other trials. Reproducibility was defined as the integral of the center SAC peak normalized by firing rate $(m)$, to account for the effect of chance (Brette, 2012):

$$
\text { reproducibility }=\frac{\int_{- \text {window }}^{+ \text {window }}\left(S A C-m^{2}\right) d t}{m},
$$

where SAC is the SAC, which is here integrated over a time range ( \pm window) centered at 0 lag, after normalizing by subtracting the squared mean firing rate, and then dividing by the mean firing rate. SACs are used as a metric of reproducibility of firing patterns across trials (Joris et al., 2006; Brette, 2012). The window for integration is functionally equivalent to the coincidence window described in previous studies (Joris et al., 2004, 2006; Louage et al., 2004). Because the half-width of the SAC's center peak can be considered a proxy for the temporal precision of a neuron's spiking (Louage et al., 2004), we set the SAC's integration window to the median halfwidth of the SAC's center peak across neurons of each dataset. To determine this half-width, we adjusted the bin size until the mean SAC displayed a significant peak [SAC value at 0 lag was greater than 2 SD above average in the flanks ( $\pm 20-$ to $50-\mathrm{ms}$ lag)] for each dataset. All bin sizes tested (0.05-20 ms; Joris et al., 2006) displayed significant peaks for ICcl while OT required a bin size of at least $2 \mathrm{~ms}$. Half-width was therefore calculated using $100-\mu$ s bin size for ICcl and $2 \mathrm{~ms}$ for OT (median half-width: ICcl dichotic = $1 \mathrm{~ms}$, ICcl free-field $=1 \mathrm{~ms}$, OT free-field $=10 \mathrm{~ms}$ ). Python code for this analysis was provided by Romain Brette (Brette, 2012).

For ICcl, reproducibility and firing rate were assessed for 6-10 different ITD and ILD values for dichotic stimulation, or a similar number of speakers for free-field stimulation, including those inducing maximal responses and points along the dynamic range of tuning curves. For OT, reproducibility was measured across speakers in the entire free-field array.

\section{Spike train distance}

As a complementary metric to the SAC analysis, the patterning of responses was assessed using a spike-train distance calculation, simply referred to as distance. Distance calculations measure the cost of transforming one spike train into another as described by Victor and Purpura (1996). This metric uses three different elementary transformations to modify spike trains: (1) insert a spike, (2) remove a spike, or (3) time shift a spike. The cost of inserting or removing a spike is assigned a value of 1 ; while shifting a spike a given amount of time ( $\Delta t \mathrm{sec}-$ onds) is assigned a cost of $q|\Delta t|$, where $q$ is a unitless scaling factor inversely related to precision (Victor, 2005). We set $q$ to a previously used value of 100 (Machens et al., 2001). Other values of $q, 33$ (Aronov et al., 2003), 64 (Victor and Purpura, 1996), 250 (Middlebrooks et al., 1994), were also tested and did not affect the general trends reported.

Permutations of all possible elementary transformations were performed using MATLAB code (code obtained from the laboratory website of Jonathan Victor at Cornell Medical School; http://www-users.med.cornell.edu/ jdvicto/pubalgor. $\mathrm{html})$, the solution which displayed the smallest final cost was then selected, and this cost was reported. Distance 
was calculated for the same data used for the SAC analysis. Here, we compared the distance between spike trains for each stimulus trial in a pairwise manner and averaged all values to obtain a final distance for each ITD, ILD, and free-field direction of each neuron.

The distance metric is zero for empty spike trains (Victor and Purpura, 1996). Thus, we omitted trials where neurons did not fire any spikes to prevent them from biasing results.

In addition, average distance increases with firing rate. To ensure that firing rate was not underlying stimulus-dependent changes in distance, we generated a correction function. Pairs of random spike trains were generated by selecting $n$ spike times from a uniform distribution over intervals matching the stimulus durations used in our study. Distance as a function of firing rate was estimated by varying the value of $n$. To take into account the across-trial variability in firing rate observed in the data, disparities of as much as twice the firing rate differences between the two model trains were allowed. To select the function that best fit the relationship between mean firing rate and distance between model spike trains, two observations were considered. First, the data displayed a nonlinear relationship (Fig. 2), and therefore, a simple linear regression would not suffice. Additionally, because the distance between trials with zero spikes will be zero by construction of this metric, when fitting the function, the $y$-intercept was forced to zero (MATLAB code polyfitZero obtained from MATLAB Central, written by Mark Mikofski; https://www.mathworks. $\mathrm{com} /$ matlabcentral/fileexchange/35401-polyfitzero). A fourth order polynomial was necessary to ensure that residuals were distributed normally, indicating this function was tracking the data properly. The curve was fitted using the range of firing rates observed by the data ( $0-51.5$ maximum spike count; Fig. 2). Because stimulus duration limits the distance metric and it varied across datasets (200 and $400 \mathrm{~ms}$ for dichotic and free field stimulation in ICcl, respectively, and $100 \mathrm{~ms}$ for OT, onset time omitted), the expected relationship between distance and spike count was estimated separately for a range of stimulus durations. The increase in distance with spike count predicted by chance was:

1. for a 100-ms stimulus:

$$
\begin{aligned}
\text { expected distance }= & \left(-1.19 \times 10^{-5}\right) x^{4}+\left(1.30 \times 10^{-3}\right) x^{3} \\
& -0.05 x^{2}+1.38 x
\end{aligned}
$$

2. for a 200-ms stimulus:

$$
\begin{aligned}
\text { expected distance }= & \left(-1.03 \times 10^{-5}\right) x^{4}+\left(1.30 \times 10^{-3}\right) x^{3} \\
& -0.06 x^{2}+1.70 x
\end{aligned}
$$

3. for a 400-ms stimulus:

$$
\begin{aligned}
\text { expected distance }= & \left(-5.62 \times 10^{-6}\right) x^{4}+\left(7.74 \times 10^{-4}\right) x^{3} \\
& -0.04 x^{2}+1.82 x
\end{aligned}
$$

where $x$ is the geometric mean (GM) firing rate between the two spike trains (spikes/second). Distances for actual
Distance correction function for ICcl free-field (400ms duration)

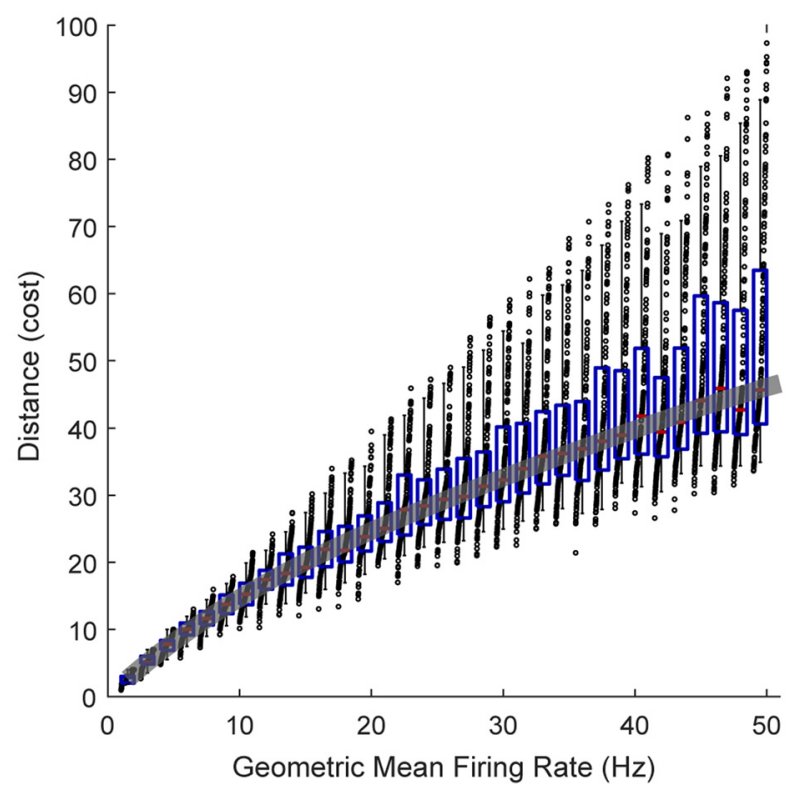

Figure 2. Effect of firing rate on spike-distance metric. Model spike trains with random spike timing were generated and their similarity measured using the spike-time distance metric described by (Victor and Purpura, 1996). GM firing rates were grouped in increments of 1.5 spikes per second. A fourth order polynomial was fit to the median values at varying joint firing rates. This function (thick black line) was then used to correct all data by the cost at chance levels. (boxplots: red line $=$ median, blue box $=$ first and third quartile, black whisker $=5 \%$ and $95 \%$, dots are sorted data for each group.) A total of 20,000 simulations were run.

data were then corrected by subtracting the values expected by chance, given the corresponding stimulus duration. Thus, negative values indicate the spike trains are more similar than expected by chance.

Distance values displayed notable outliers (detected using median absolute deviation methods) which made the distribution of distances across the population heteroscedastic (variability was not constant across firing rate). Because Pearson correlation makes the assumption of homoscedasticity and that there are no outliers, we used Spearman rank-order correlation coefficients to evaluate the relationship between distance and firing rate.

\section{Spike-time synchrony of space-map cells}

Synchrony between simultaneously recorded OT neurons stimulated in free-field was assessed using previously described methods. In brief, cross-correlograms (CCGs) of pairs of simultaneously recorded neurons were computed for sound emitted by speakers in different locations. As for reproducibility and distance computations, spikes within the onset of the stimulus presentation (first $50 \mathrm{~ms}$ ) were omitted from the analysis. Spikes within $1 \mathrm{~ms}$ of one another were deemed coincident. CCGs were smoothed using a 5-ms sliding window (Bair et al., 2001; Kohn and Smith, 2005). Adding up all coincidences yielded a standardCCG. To determine whether reproducible firing across 
trials influenced this synchrony, a shifted-CCG (Bair et al., 2001) was also computed. The shifted-CCG measures the number of coincident spikes between two neurons across non-simultaneous trials of identical sounds, specifically counting coincidences from trial $n$ of neuron 1 and trial $n+1$ of neuron 2. This CCG captures the amount of coincidences that may be due to spiking patterns driven by temporal dynamics of the stimulus. Here, we used the shiftedCCG to investigate whether synchrony of nearby cells could be explained by patterned activity of these cells. To evaluate coincidences not related to temporal dynamics of the stimulus, the shifted-CCG was subtracted from the standardCCG for each neuron (coincidences for each time lag bin in the shifted-CCG were removed from the matching bin in the standard-CCG), to produce a corrected-CCG. To take into account coincidences occurring due to chance alone related to firing rate and bin size, CCGs were normalized by the bin size multiplied by the GM firing rate (square root of the product of firing rates of the two neurons), as in other studies (Bair et al., 2001). Synchrony was quantified by the integral of the CCG's center peak. A standard boundary was selected by averaging the CCG of all neurons and identifying a range which fit this large center peak. For this dataset the boundary was $\pm 10 \mathrm{~ms}$. Sound locations that did not elicit sufficient spiking ( $<2 \mathrm{SD}$ above the mean baseline) for both neurons in a pair were omitted from the calculation of synchrony. In addition, pairs needed to display sufficient spiking for at least two speakers to be included in the dataset (189/194 pairs fit criterion).

To assess how synchrony varied with positions in space, GM was calculated for the response of pairs of neurons to each speaker location. The GM firing rate is a standard metric for assessing the combined firing rate of neurons (Bair et al., 2001; Kohn and Smith, 2005; Smith and Kohn, 2008).

\section{Up-and-down states}

To investigate whether up-and-down states induced by anesthesia underlay spiking co-variation, we used a method described by Pachitariu et al. (2015) to detect upstates based on spike counts. The spike times of all units recorded by a single tetrode were pooled to attain a measurement of multiunit activity (MUA) during baseline recordings, in the absence of sound stimulation. Spike times were binned in 10-ms intervals to create a running spike count. A 10-bin wide median filter (custom-written MATLAB routine, but see function medfilt1) was applied to the spike count, where each value was replaced by the median spike count of the 10 neighboring bins (edges were omitted). Up-and-down states are generally hundreds to thousands of milliseconds long (Parga and Abbott, 2007; McFarland et al., 2011; Bragin et al., 2012; Xu and Wang, 2014), therefore this filter window was sufficient to identify potential up-states. Bouts of firing across more than half of the bins of the median filter window (six or more in 10) yielded median values above 0 , the criterion used by this method to define up-states.

\section{Correlation analysis}

The Pearson product-moment correlation coefficient $(r)$ was calculated to describe the relationship between reproducibility and distance with mean firing rate of each neuron, and the same was done for synchrony and the GM firing rate for each pair of neurons. The $r$ values were transformed using Fisher's z-transformation for statistical tests and then converted back to $r$ s for reporting. Normalized reproducibility and mean firing rates were also pooled and tested for overall correlation, and the same was done for synchrony and GM firing rate. The significance of the correlation was tested using a two-tailed $t$ test.

\section{Computational model}

We used a model to investigate how stimulus dependent spiking patterns in ICcl could affect synchrony between nearby neurons in OT. The input to $\mathrm{ICcl}$ was modeled using a previously described model of the auditory periphery in the barn owl (Fischer et al., 2009). Briefly, broadband Gaussian noise signals between 0.2 and $12 \mathrm{kHz}$ were passed through a gammatone filter-bank with center frequencies ranging from 1 to $9 \mathrm{kHz}$ in $0.2-\mathrm{kHz}$ steps. The time constants of the filters were specific to the owl and estimated from Köppl (1997) to model cochlear filters. The outputs of the cochlear filters were crosscorrelated in each frequency channel. The cross-correlation model included a gain-control component so that the amplitude of the cross-correlation output grows with stimulus level in a manner that matches the relationship between stimulus level and firing rate in barn owl coincidence detector neurons (Fischer et al., 2009).

ICcl neurons were modeled as adaptive exponential leaky integrate-and-fire neurons that were driven by the output of the cross-correlation model. The adaptive exponential leaky integrate-and-fire model has been shown to be a good model of intracellular responses in the barn owls' midbrain (Fontaine et al., 2014b). The subthreshold membrane potential of the ith ICcl neuron obeyed the differential equation:

$$
\begin{aligned}
\tau \frac{d V_{i}(t)}{d t}=\left(V_{L}\right. & \left.-V_{i}(t)\right)+\Delta \exp \left(\frac{V_{i}(t)-\theta_{i}(t)}{\Delta}\right) \\
& +g_{i}(t)\left(V_{E}-V_{i}(t)\right),
\end{aligned}
$$

where the membrane time constant $\tau=60 \mathrm{~ms}$, the leak reversal potential $V_{L}=-75 \mathrm{mV}$, the synaptic reversal potential [dot]VE $=0 \mathrm{mV}, \theta_{i}(t)$ is the dynamic spiking threshold, $\Delta=1 \mathrm{mV}$, and $g_{i}(t)$ is the input conductance. Note that the input conductance $g_{i}(t)$ is normalized by the leak conductance, and therefore $g_{i}(t)$ has no units. The input conductance is a function of the output of the crosscorrelation model at the best ITD and best frequency of the $i$ th ICcl neuron, which is denoted $\xi(t)$ The cross-correlation output was transformed using an exponential nonlinearity followed by a linear rectification to generate the input signals to the modeled ICcl cells:

$$
s_{i}(t)=a\left[\exp \left(x_{i}(t)\right)-b_{i}\right]_{+},
$$

where $b_{i}$ is the average value of $\exp (\xi(t))$ and $a=45$. The input conductance $g_{i}(t)=s_{i}(t)+n_{i}(t)$ is a sum of $s_{i}(t)$ and a neuron-specific noise signal $n_{i}(t)$ The noise $n_{i}(t)$ was a 
lowpass Gaussian noise signal with flat amplitude spectrum up to $1 \mathrm{kHz}$. The root mean square (RMS) level of the noise was proportional to the square root of the RMS level of the signal $s_{i}(t)$ so that the signal-to-noise ratio grows as the square root of the RMS level of the signal. A neuronspecific noise signal $n_{i}(t)$ was included to model differences in spike-train reproducibility between neurons, and therefore the noise signals were independent across neurons.

The dynamic spiking threshold obeyed the differential equation

$$
\tau_{\theta} \frac{d \theta_{i}(t)}{d t}=V_{1}+k_{1} \ln \left(1+\exp \left(\frac{V_{i}(t)-V_{2}}{k_{2}}\right)\right),
$$

where $\tau_{\theta}=5 \mathrm{~ms}, V_{1}=-57 \mathrm{mV}, k_{1}=5 \mathrm{mV}, k_{2}=1 \mathrm{mV}$, and $V_{2}=-67 \mathrm{mV}$. A spike occurs when the membrane potential exceeds the dynamic threshold $\theta_{i}(t)$, after which the membrane potential is reset to $-55 \mathrm{mV}$ and held constant for a refractory period of $1 \mathrm{~ms}$.

The ICcl spikes formed the input to a population of space-map neurons. The subthreshold membrane potential of the ith space-map neuron obeyed the differential equation:

$$
\begin{aligned}
\tau \frac{d V_{i}(t)}{d t} & =\left(V_{L}-V_{i}(t)\right)+\Delta \exp \left(\frac{V_{i}(t)-\theta_{i}(t)}{\Delta}\right) \\
& +\sum_{j=1}^{N} \omega_{i j} g_{\text {syn }, j}(t)\left(V_{\text {syn }, j}-V_{i}(t)\right)
\end{aligned}
$$

where $\omega_{i j}$ is a connection weight, $g_{s y n, j}(t)$ is the synaptic conductance due to the response of the $j$ th input neuron, $N=2665$ is the number of input ICcl neurons, and the synaptic reversal potential $V_{\text {syn, }}$ is equal to $0 \mathrm{mV}$ for excitatory synapses and $-80 \mathrm{mV}$ for inhibitory synapses. The parameters $\tau, V_{L}$, and $\Delta$ of the membrane potential and the parameters of the dynamic spiking threshold for the spacemap neurons were the same as described for the $\mathrm{ICCl}$ neurons. The connection weights varied with the difference in the best ITDs and best frequencies of the ICcl and space-map neurons, $\omega_{i j} \propto \omega_{I T D, i j} \times \omega_{F, i j}$. The pattern of the ITD-dependent component of the weights was given by the difference between a central Gaussian centered at best ITD of the space map neuron and two Gaussians centered at ITDs that differed from the best ITD by one half of the period of the neuron's best frequency:

$$
\begin{aligned}
\omega_{I T D, j i}= & \exp \left(-\frac{1}{2}\left(\frac{I T D_{i}-I T D_{j}}{\sigma}\right)^{2}\right) \\
& -\frac{1}{2} \exp \left(-\frac{1}{2}\left(\frac{I T D_{i}+0.5 P_{i}-I T D_{j}}{\sigma}\right)^{2}\right) \\
& -\frac{1}{2} \exp \left(-\frac{1}{2}\left(\frac{I T D_{i}-0.5 P_{i}-I T D_{j}}{\sigma}\right)^{2}\right)
\end{aligned}
$$

where $I T D_{i}$ is the best ITD of the space-map neuron, $I T D_{j}$ is the best ITD of the jth ICcl neuron, $P_{i}$ is the period of the space-map neuron's best frequency, and $\sigma=10 \mu \mathrm{s}$. The pattern of the frequency-dependent component of the weights was given by a Gaussian centered at best frequency of the space map neuron $\omega_{F, i j}=\exp \left(-\frac{1}{2}\left(\frac{B F_{i}-B F_{j}}{\sigma}\right)^{2}\right)$, where $B F_{i}$ is the best frequency of the space-map neuron, $B F_{j}$ is the best frequency of the jth ICcl neuron, and $\sigma=$ $6500-20 \times I T D_{i} \mathrm{~Hz}$. The connection weight $\omega_{i j}$ was the magnitude of the product of these values, while its sign determined whether the input was excitatory or inhibitory. For excitatory inputs the synaptic reversal potential was $V_{s y n, j}=0 \mathrm{mV}$ and for inhibitory inputs the synaptic reversal potential was $V_{s y n, j}=$ $80 \mathrm{mV}$. The synaptic conductance was an exponential: $g_{s y n, j}(t)=\sum_{n} \exp \left(-\frac{t-t_{j, n}}{\tau_{s}}\right) H\left(t-t_{j, n}\right)$, where $t_{j, n}$ is the time of the $n$th spike of the $j$ th $\mathrm{ICcl}$ neuron and $H(\mathrm{t})$ is the unit step function. The time constant of the synaptic conductance was $\tau_{s}=5 \mathrm{~ms}$.

We modeled a population of neurons downstream from the auditory space map to investigate the effects of spiking synchrony in the space map on the coding of ITD. The subthreshold membrane potential of the ith output neuron was modeled using the same form of model as used for the space map:

$$
\begin{aligned}
\tau \frac{d V_{i}(t)}{d t} & =\left(V_{L}-V_{i}(t)\right)+\Delta \exp \left(\frac{V_{i}(t)-\theta_{i}(t)}{\Delta}\right) \\
& +\sum_{j=1}^{N} \omega_{i j} g_{\text {syn. } j}(t)\left(V_{\text {syn } . j}-V_{i}(t)\right)
\end{aligned}
$$

but here $g_{s y n, j}(t)$ is the synaptic conductance from the $j$ th space map neuron and $N=81$ is the number of input space-map neurons. The connection weights between neurons in the input and output layers of the model followed a Gaussian-shaped function with a SD of $30^{\circ}$. The SD accounted for the population in the input layer that converged onto the output layer. The results were robust to changes in the SD over the range $5-75^{\circ}$.

Synchrony in the modeled populations was quantified with the same types of CCGs used for space-map neurons (described in section Spike-time synchrony of space-map cells above). The effect of stimulus-dependent synchrony in the space-map population was assessed by the suppression of side peaks forming in the pattern of spike counts produced by the output population when plotted as a function of the preferred ITD. We first measured the side peaks in the output population for the original model data displaying stimulus-dependent synchrony. We next altered the synchrony in the side peak by shifting spike times of neurons in the side peak. To manipulate synchrony in side peaks by shifting spike times, the stimulus interval was first divided into 25 -ms segments. The average spike time within side peaks was calculated for each 25-ms segment across modeled neurons, to create a template spike timing. In each $25-\mathrm{ms}$ segment, spike times were shifted toward these template spike times. The amount of shift toward the mean time was controlled 


\section{Example ICcl Neuron - Dichotic}

A

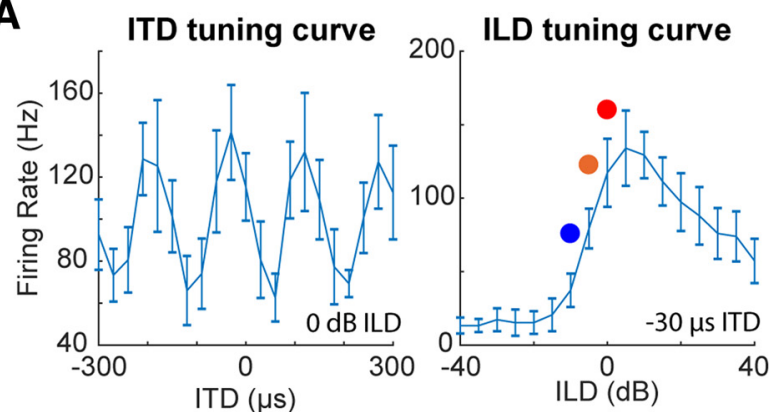

B

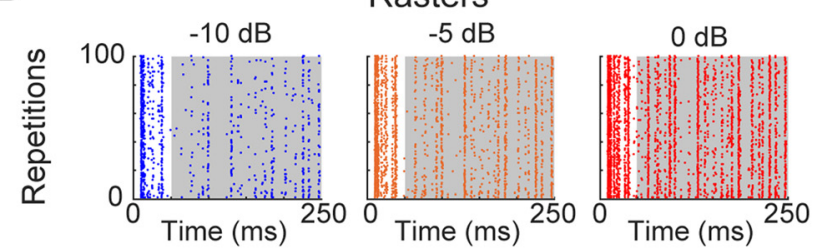

C

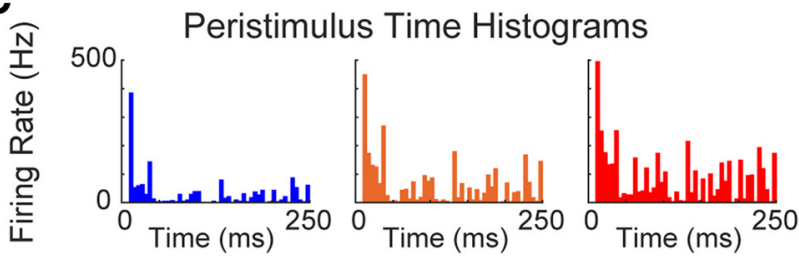

D

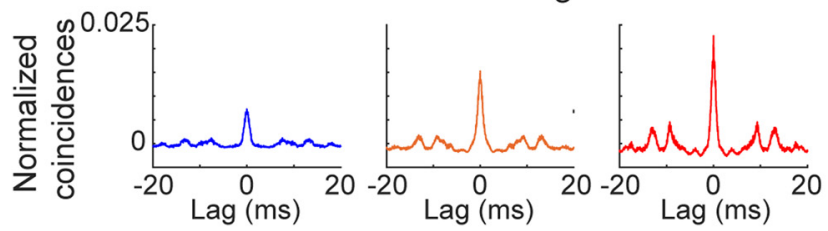

Figure 3. Reproducibility in ICcl to varying binaural cues. $\boldsymbol{A}$, Tuning curves of an example ICcl neuron for ITD (left) and ILD (right). Positive ITDs and ILDs indicate, respectively, sounds leading and higher intensity on the right ear (contralateral side for the example cell). Colored dots in the ILD tuning curve are the examples displayed in $\boldsymbol{B}-\boldsymbol{D}$ (plots represent mean \pm SEM, $n=10$ repetitions per trial). $\boldsymbol{B}$, Example spiking raster plots of neuron shown in $\boldsymbol{A}$ at varying ILDs (indicated on top of each plot). Gray background represents region of stimulation used for SAC and distance analysis (50-ms onset omitted). C, Peristimulus time histograms generated from the raster plots in $\boldsymbol{B}$ (5-ms bin size). $\boldsymbol{D}$, SACs generated from the rasters in $\boldsymbol{B}$.

to produce synchrony in the side peak that matched the synchrony in the main peak. This manipulation did not change the overall firing rate of the neurons. We then compared the side peak in the output population generated from this new space-map population where synchrony is high in the side peak.

For calculating reproducibility and synchrony in modeled neurons, the first $50 \mathrm{~ms}$ of the onset was omitted to match the analysis of neural data.

\section{Statistical analysis}

All datasets were tested for normalcy (D'AgostinoPearson normality test) using GraphPad Prism 7. Nonparametric tests were used when at least one group was not normally distributed. Statistical tests used are listed in the results. Correlation coefficients were computed using MATLAB. Quantification of data are written as mean \pm SD unless otherwise noted.

\section{Code accessibility}

Relevant code for calculation of reproducibility, spiketime distance, spike-time synchrony, and model neuron populations are publicly available through GitHub at https://github.com/penalab/Beckert-et-al.-2020.

\section{Results}

To assess the precision of spiking patterns for sounds from different locations, we measured across-trial reproducibility in single units recorded from the lateral shell of the central nucleus of the inferior colliculus (ICcl; $n=68$ units). Reproducibility was quantified by computing the integral of the central peak of the SAC, a metric of the similarity and temporal precision of spike timing across trials of identical (frozen) broadband noise (Joris et al., 2006; Brette, 2012), and the across-trial spike distance (Victor and Purpura, 1996). Stimuli were presented either through earphones (varying binaural spatial cues) or in free-field (varying spatial location). The analysis of reproducibility in ICcl was followed by tetrode recordings in the downstream map-of-space in the OT $(n=93$ units from 20 recording sites, resulting in 194 pairs of simultaneously recorded neurons). Across-trial reproducibility and distance, as well as spike-time synchrony, were also examined in OT neurons. Finally, the underlying network mechanisms and coding implications of these findings were assessed through computational modeling.

\section{Reproducibility in ICcl depends on binaural cues}

Single units in $\mathrm{ICCl}$ displayed tuning to the primary binaural cues for sound localization, ITD and ILD, as previously described (Keller and Takahashi, 2000; Fischer et al., 2007; Singheiser et al., 2012). In particular, rate-ITD curves of ICcl neurons were quasi-periodic with a larger center peak but prominent side peaks, and rate-ILD curves displayed sigmoidal or open peak shapes favoring higher sound level on the contralateral side (Fig. 3A).

Neurons displayed reproducible firing across trials, resulting in patterned spike rasters (Fig. 3B), peaks in poststimulus time histograms (Fig. $3 C$ ), and large peaks at 0 delay in the SACs (Fig. 3D). This is consistent with previous reports that ICcl neurons exhibit reproducible spike timing across trials of identical sounds (Keller and Takahashi, 2000).

The reproducibility of the spike patterns was measured at different ITD and ILD values. The relationship between reproducibility and firing rate was assessed with correlation analysis for each individual neuron and across the sample (grouping all points for all neurons). To account for variability across neurons, firing rates and 
Reproducibiliy in ICcl varying ITD and ILD
A Reproducibility

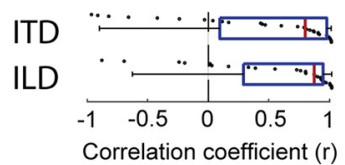

$B-r=0.44743 ; p<0.0001$
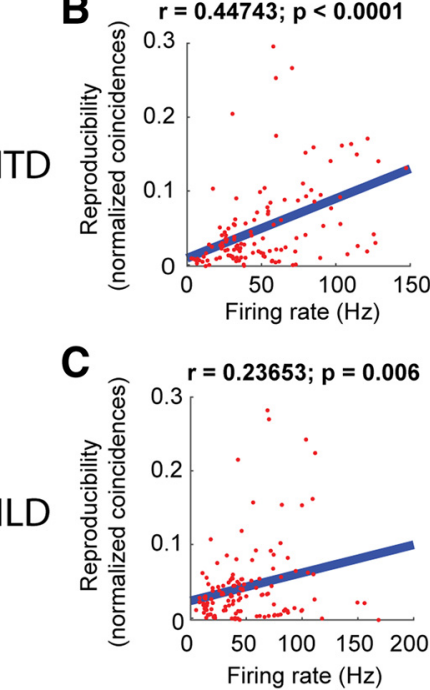

D Distance

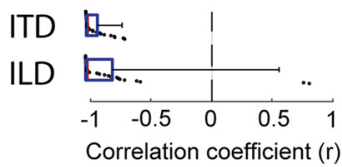

E $r_{s}=-0.91798 ; p<0.0001$

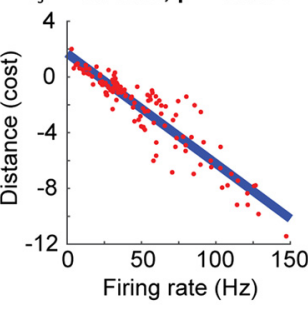

F $\quad r_{s}=-0.88892 ; p<0.0001$

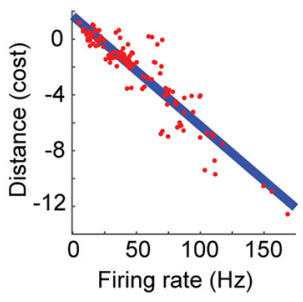

Figure 4. Relationship between reproducibility and firing rate of ICcl neurons $(N=33)$, varying binaural cues. $\boldsymbol{A}$, Correlation coefficients $(r)$ of reproducibility against firing rate in each neuron for varying ITD (top) and ILD (bottom). Red lines indicate the median, blue boxes represent the first and third quartile, and whiskers are the 5th and 95th percentiles. B, C, Reproducibility against firing rate for all neurons for varying ITD $(\boldsymbol{B})$ and ILD $(\boldsymbol{C})$. Red dots represent individual data points and blue lines are linear regressions. Correlation coefficients $(r)$ and $p$ values are shown above each plot. $\boldsymbol{D}-\boldsymbol{F}$, Same description as for $\boldsymbol{A}-\boldsymbol{C}$ but for the corrected Victor-Purpura distance metric.

reproducibility were normalized to the maximum for each cell. Reproducibility was significantly correlated with firing rate at varying ITD and ILD. The mean correlation coefficient for individual neurons $(n=33)$ was significantly nonzero (ITD: mean $r=0.45 \pm 0.63 ; t=3.68 ; p=0.0009$; twotailed one-sample $t$ test run on $z$ values; ILD: mean $r=$ $0.57 \pm 0.53 ; t=5.72 ; p<0.0001$; two-tailed one-sample $t$ test run on $z$ values; Fig. $4 A$ ). This correlation was also significant after pooling responses across neurons (ITD: $r=0.45 ; p<0.0001$; ILD: $r=0.24 ; p=0.006$; two-tailed $t$ test; Fig. $4 B, C$ ). SACs were normalized by mean firing rate (Joris et al., 2006; Brette, 2012), which accounts for increases in SAC height due to spikes occurring within the same bin due to chance alone. The relationship between response strength and reproducibility indicates an enhancement of patterned spiking evoked by sounds at the neurons' preferred binaural cues.

As a complementary approach, we assessed the similarity of spiking across trials using a cost-distance metric (Victor and Purpura, 1996). A correction factor was applied to take into account the anticipated effect of
Relationship between reproducibility and firing rate are different when varying ITD and ILD

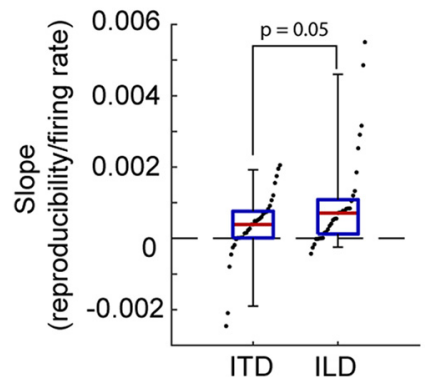

B

Dynamic range of firing rates when varying ITD and ILD per neuron

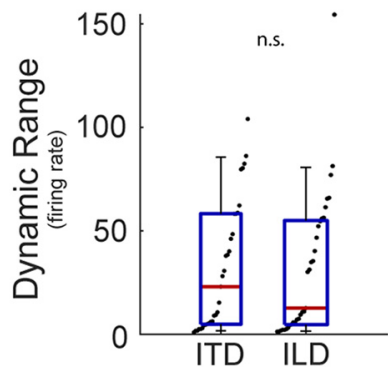

Figure 5. Relationship between reproducibility and response strength depends on the type of binaural cue. $\boldsymbol{A}$, Comparison of slopes of linear regressions computed for each neuron's reproducibility as a function of firing rate driven by varying ITD and ILDs. $\boldsymbol{B}$, Comparison of the dynamic range of firing rates evoked by varying ITD and ILD for each neuron. Red lines indicate the median, blue boxes represent the first and third quartile, and whiskers are the 5th and 95th percentiles.

increasing numbers of spikes on the distance metric (see Materials and Methods), leading to negative distance values indicating that spike patterns are more similar than what is expected by chance. Across-trial distance of spike trains in ICcl was negatively correlated with firing rate as both ITD and ILD varied. The mean correlation coefficient for individual neurons was significantly non-zero (ITD: mean $r=-0.94 \pm 0.10 ; t=13.99 ; p<0.0001$; twotailed one-sample $t$ test run on $z$ values; ILD: mean $r=$ $-0.80 \pm 0.44 ; t=8.92 ; p<0.0001$; two-tailed one-sample $t$ test run on $z$ values; Fig. $4 D$ ) and the correlation after pooling across neurons was highly significant (Spearman correlation: ITD: $r_{\mathrm{s}}=-0.92$; ILD: $r_{\mathrm{s}}=-0.89$; both $p<0.0001$; two-tailed $t$ test; Fig. $4 E, F$ ). This negative correlation between across-trial distance and firing rate is consistent with the positive correlation between reproducibility and firing rate reported in Fig. 4 above. These results indicate that the spike-timing reproducibility increases with response strength in ICcl neurons.

To assess whether reproducibility could simply be predicted by firing rate, we compared the relationship between reproducibility and firing rate when ITD and ILD were independently changed. For each neuron, a linear regression for reproducibility as a function of firing rate when either ITD or ILD was changed was calculated. We then compared the slopes for each regression. The slopes when ITD was varied (mean slope: $0.0004 \pm 0.0009$ reproducibility/firing rate units) were moderately shallower than for ILD (mean slope: $0.0010 \pm 0.0014$ reproducibility/firing rate; $p=0.05$; two-tailed Wilcoxon signed-rank; Fig. $5 A$ ). We note that the dynamic range of firing rate elicited by ITD and ILD variations was not significantly different across neurons $(p=0.91$; two-tailed paired $t$ test; Fig. $5 B)$, so this difference is not related to the overall number of spikes. This further suggests that while there is a relationship between reproducibility and firing rate for 


\section{Example ICcl Neuron - Free-field}

A

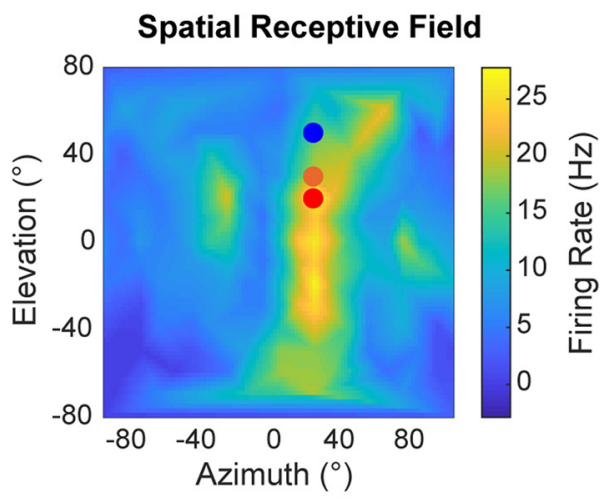

B

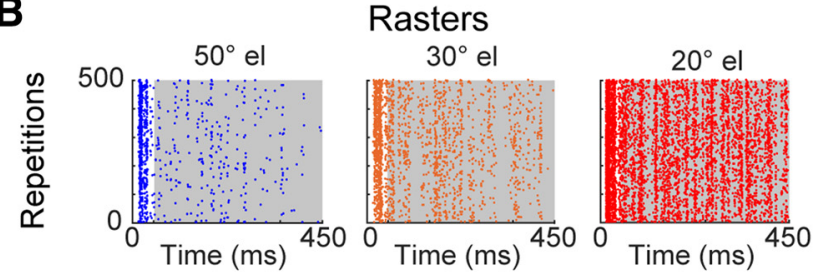

C

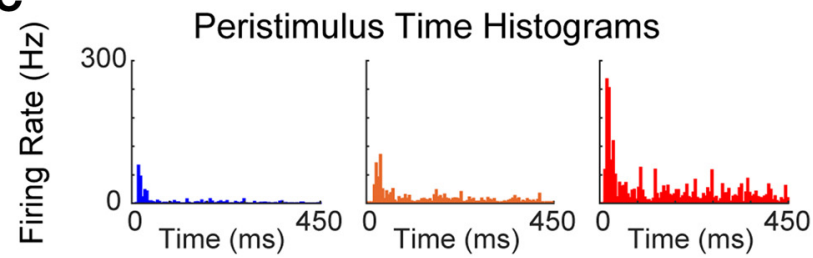

D

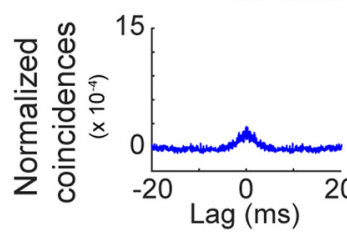

Shuffled Autocorrelograms

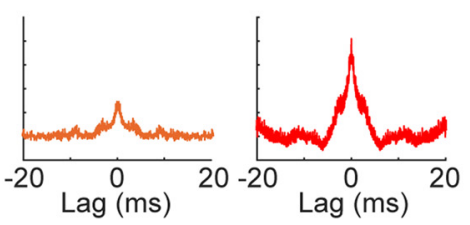

Figure 6. Reproducibility in $\mathrm{ICcl}$ to sounds from varying positions in auditory space. $\boldsymbol{A}$, Example SpRF for an ICcl neuron. Colored dots indicate responses displayed in $\boldsymbol{B}-\boldsymbol{D}$. $\boldsymbol{B}$, Spiking raster plots at varying elevations, from the example cell shown in $\boldsymbol{A}$. Gray background represents region of stimulation used for SAC and distance analysis (50-ms onset responses not considered in the analysis). $\mathbf{C}$, Peristimulus time histograms generated from the raster plots in $\boldsymbol{B}$ (5-ms bin size). $\boldsymbol{D}$, SACs generated from the rasters in $\boldsymbol{B}$.

varying ITD and ILD, the rate at which they change may differ and be therefore stimulus-dependent rather than solely rate dependent.

\section{Stimulus-dependent reproducibility in ICcl for sounds in free-field}

Dichotic stimulation allows the precise manipulation of individual stimulus parameters, bypassing the filtering properties of the head, described by the head-related

\section{Reproducibiliy in ICcl varying sound directions}

\section{A Reproducibility

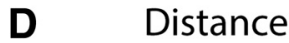

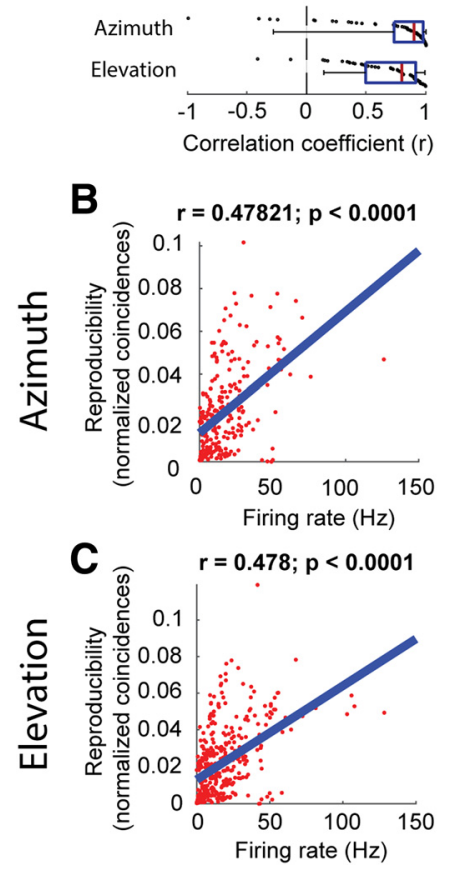

$\mathbf{E}$
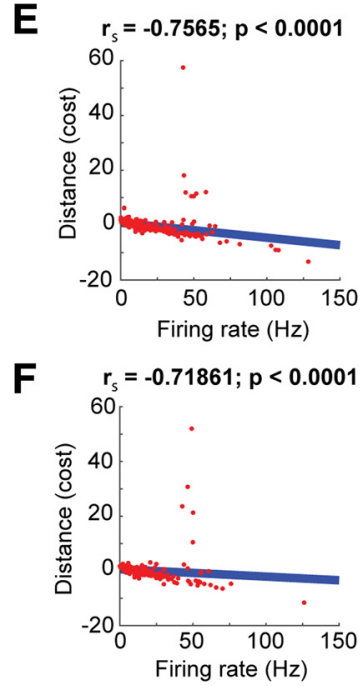

Figure 7. Relationship between reproducibility and firing rate in ICcl $(N=64)$, varying spatial location in free field (same format as in Fig. 4). $\boldsymbol{A}$, Correlation coefficients $(r)$ of reproducibility against firing rate for each neuron for varying azimuth (top) and elevation (bottom). $\boldsymbol{B}, \boldsymbol{C}$, Reproducibility as a function of firing rate for all neurons for varying azimuth $(\boldsymbol{B})$ and elevation $(\boldsymbol{C})$. Firing rates and reproducibility are normalized by the maximum and minimum for each neuron. $\boldsymbol{D}-\boldsymbol{F}$, Same description as for $\boldsymbol{A}-$ $\boldsymbol{C}$ but for the corrected Victor-Purpura distance metric.

transfer function (HRTF). However, these filtering properties modulate sounds in a direction-dependent manner (Keller et al., 1998). Therefore, to test the relationship between sound direction and reproducibility in a more naturalistic setting preserving the natural modulation of the envelope with direction, ICcl responses were also examined using stimuli presented in free-field. ICcl neurons displayed welldelimited spatial tuning (Fig. $6 A$ ) and reproducible firing patterns for frozen noise in free-field (Fig. 6B-D).

Reproducibility was highest at the center of the SpRF and decreased away from the center in both azimuthal and elevational directions, displaying significant positive correlation to firing rate in individual neurons (azimuth: mean $r=0.72 \pm 0.41 ; t=9.17 ; p<0.0001 ; n=64$; twotailed one-sample $t$ test run on $z$ values; elevation: mean $r=0.70 \pm 0.30 ; t=11.68 ; p<0.0001 ; n=64$; two-tailed one-sample $t$ test run on $z$ values; Fig. 7A). This relationship held in the pooled data (Pearson correlation: azimuth: $r=0.48$; elevation: $r=0.48$; both $p<0.0001$; two-tailed $t$ test; Fig. $7 B, C)$. Similarly, across-trial distance was negatively correlated with firing rate changes induced by varying sound direction, with significant correlations for individual neurons (azimuth: mean $r=-0.88 \pm 0.19$; $p<0.0001 ; n=64$; two-tailed Wilcoxon signed-rank test 
run on $z$ values; elevation: mean $r=-0.85 \pm 0.32$; $p<0.0001 ; n=64$; two-tailed Wilcoxon signed-rank test run on $z$ values; Fig. 7D) and for pooled data (Spearman correlation: azimuth $r_{\mathrm{s}}=-0.76$; elevation $r_{\mathrm{s}}=-0.72$; both $p<0.0001$; two-tailed $t$ test; Fig. 7E, $F$ ). These results further demonstrate that reproducibility in $\mathrm{ICcl}$ increases with firing rate.

Taken together, the across-trial reproducibility in $\mathrm{ICcl}$ was dependent on the firing rate elicited by changes in binaural cues and sound direction. These results show that sound location affects the reproducibility of spike timing across trials in ICcl.

\section{Reproducibility in OT}

We next explored stimulus-dependent reproducibility in the downstream area OT, which contains a map of auditory space (Knudsen, 1982) and commands head-orienting behavior (du Lac and Knudsen, 1990). We recorded deep-layer OT neurons with tetrodes and stimulated with sounds presented in free field, which allowed assessing the population response of local clusters of OT neurons to naturalistic sounds.

Previous reports on neurons in the midbrain map of auditory space showed phase-locking to the sound's envelope using sinusoidal amplitude-modulated sound, which has a very robust envelope (Keller and Takahashi, 2005; Nelson and Takahashi, 2010). Indeed, tracking such strong amplitude modulations has been hypothesized to be important for differentiating nearby sound sources and suppressing responses to echoes (Keller and Takahashi, 2005; Nelson and Takahashi, 2010; Baxter et al., 2013). However, the across-trial reproducibility and ability to lock to the envelope of stimuli without sinusoidal-amplitude modulations has not been assessed. Typically, neurons lose their ability to lock to lower-order properties of sound (such as phase and envelope) as they progress down the auditory pathways (Johnson, 1980; Winter and Palmer, 1990; Lu et al., 2001; Köppl and Carr, 2003; Joris et al., 2004; Louage et al., 2004; Christianson and Peña, 2006, 2007; Liu et al., 2006; Woolley et al., 2009; Steinberg and Peña, 2011; Kim and Doupe, 2011; Atencio et al., 2012; Calabrese and Woolley, 2015). Therefore, we anticipated that neurons in OT would display a reduced ability to lock to the envelope compared with $\mathrm{ICcl}$ neurons.

We examined whether OT neurons displayed reproducible firing patterns in response to frozen noise stimuli in the same manner as in ICcl. In fact, OT neurons $(n=93$; Fig. 8A) showed reproducible spike trains (Fig. 8B,C), indicated by defined center peaks of their SACs (Fig. 8D). However, a substantially larger integration window was required to generate SACs with defined center peaks (Materials and Methods). While the difference in integration windows did not allow a direct comparison of reproducibility values measured by SACs in ICcl and OT neurons, the larger integration window required in OT is consistent with the anticipated decrease of stimulus-induced patterning in this region compared with $\mathrm{ICcl}$. This difference in precision is expected, as envelope-locking generally weakens along auditory pathways (Lu et al.,

\section{Example OT Neuron - Free-field}

A
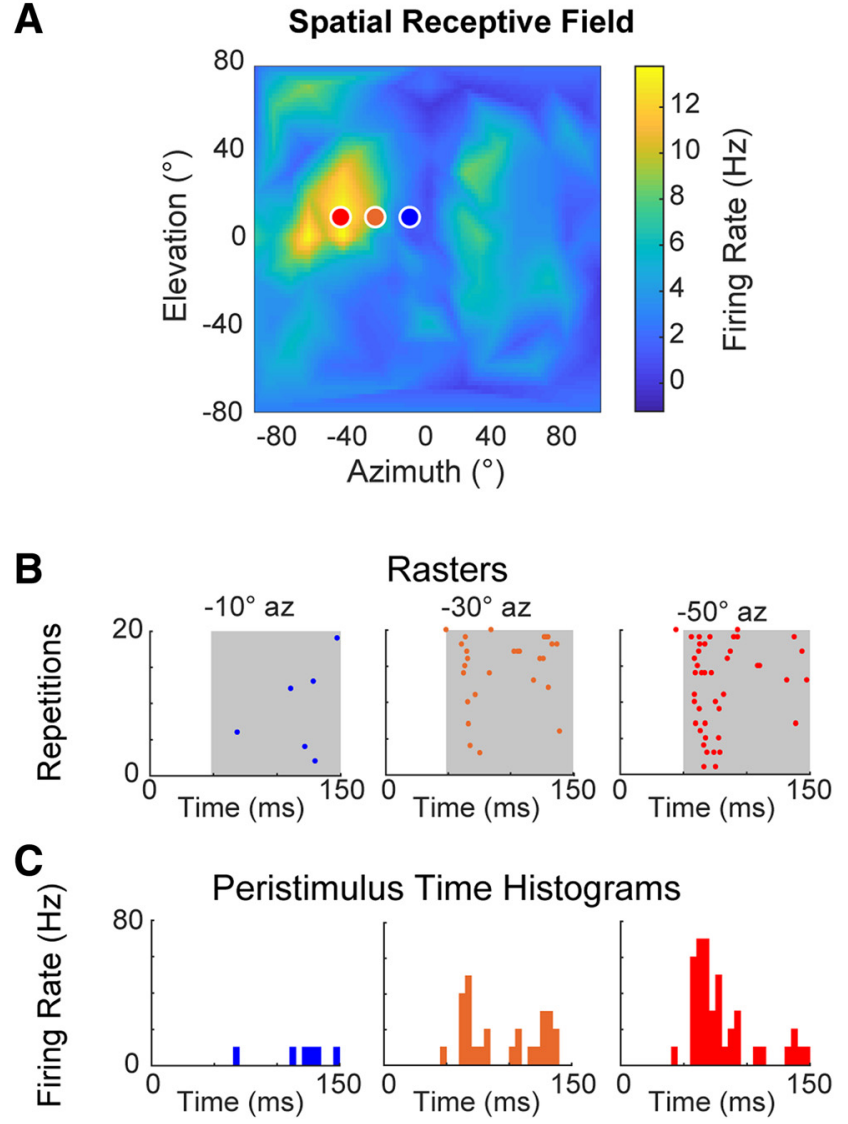

D

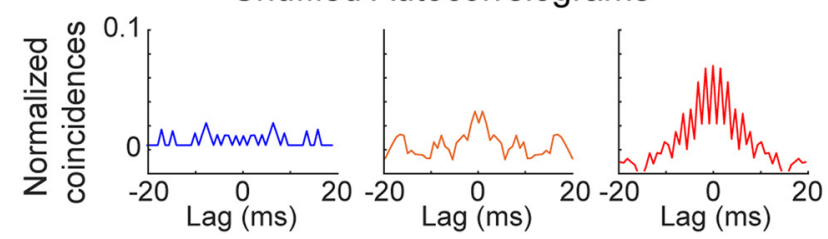

Figure 8. Reproducibility in OT to sounds from varying positions in auditory space. $\boldsymbol{A}$, Example SpRF of an OT neuron. Colored dots indicate responses displayed in $\boldsymbol{B}-\boldsymbol{D}$. $\boldsymbol{B}$, Example spiking raster plots at varying azimuth from the cell shown in $\boldsymbol{A}$. Gray background represents region of stimulation used for SAC, distance, and synchrony analysis. $\boldsymbol{C}$, Peristimulus time histograms generated from the raster plots in $\boldsymbol{B}$ (5-ms bin size). $\boldsymbol{D}$, SACs generated from the rasters in $\boldsymbol{B}$.

2001; Joris et al., 2004; Christianson and Peña, 2006, 2007; Steinberg and Peña, 2011). In line with this observation, the distance metric in OT cells was significantly higher than in ICcl (ICcl dichotic: $-3.69 \pm 3.38$, ICcl freefield: $-1.27 \pm 2.88$, OT: $1.16 \pm 0.89$ cost; Kruskal-Wallis with two-tailed Dunn's multiple comparison; $p<0.0001$ across all datasets).

Despite the reduced precision compared with $\mid \mathrm{Ccl}$, reproducibility in OT was still stimulus dependent in individual neurons (azimuth: mean $r=0.22 \pm 0.57 ; p=0.0031$; $n=64$; two-tailed Wilcoxon rank-sum test run on $z$ values; 
Reproducibiliy in OT varying sound directions
A Reproducibility
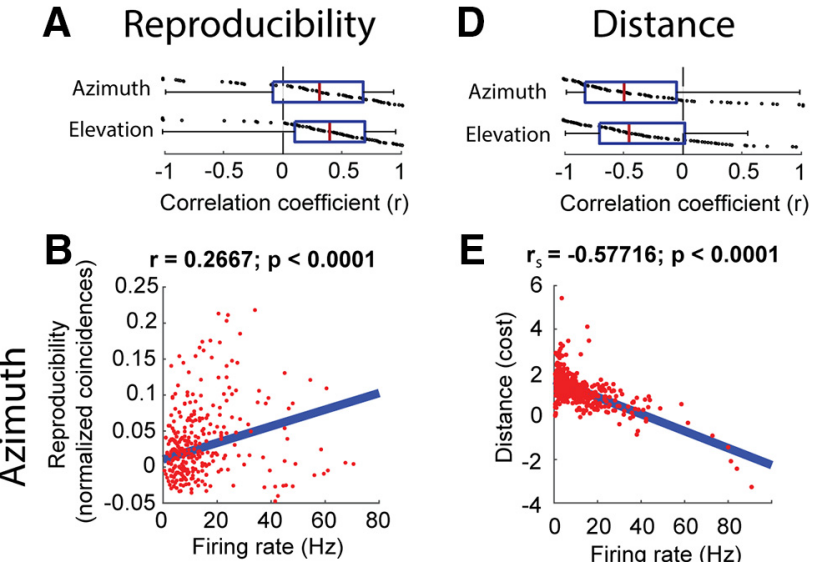

C $\quad r=0.3026 ; p<0.0001$

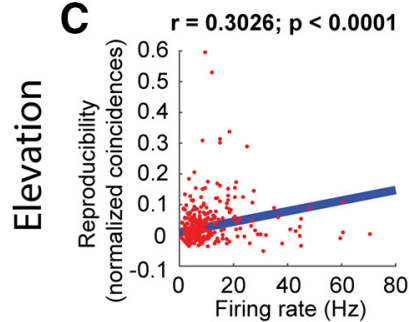

E $\quad r_{s}=-0.57716 ; p<0.0001$ 6 .

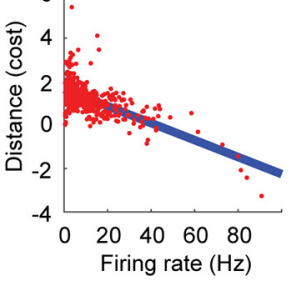

F $\quad r_{s}=-0.38595 ; p<0.0001$

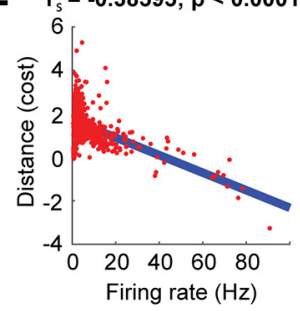

Figure 9. Relationship between reproducibility and firing rate in OT $(N=93)$ varying spatial location (same format as in Fig. 4). $\boldsymbol{A}$, Correlation coefficients $(r)$ of reproducibility against firing rate for each neuron for varying azimuth (top) and elevation (bottom). $\boldsymbol{B}, \boldsymbol{C}$, Reproducibility as a function of firing rate for all neurons while varying azimuth $(\boldsymbol{B})$ and elevation $(\boldsymbol{C})$. Firing rates and reproducibility are normalized by the maximum and minimum for each neuron. $\boldsymbol{D}-\boldsymbol{F}$, Same description as for $\boldsymbol{A}-\boldsymbol{C}$ but for the corrected Victor-Purpura distance metric.

elevation: mean $r=0.29 \pm 0.53 ; p<0.0001 ; n=64$; twotailed Wilcoxon rank-sum test run on $z$ values; Fig. $9 A$ ) as well as for pooled data points (Pearson correlation: azimuth: $r=0.27$; elevation: $r=0.30$; both $p<0.0001$; twotailed $t$ test; Fig. 9B,C). Consistently, distance was negatively correlated with response strength to varying sound direction (azimuth: mean $r=-0.32 \pm 0.57 ; p<0.0001$; $n=64$; two-tailed Wilcoxon rank-sum test run on $z$ values; elevation: mean $r=-0.33 \pm 0.49 ; p<0.0001 ; n=64$; twotailed Wilcoxon rank-sum test run on $z$ values; Fig. $9 D$ ). This finding indicates that although the precision of spiking in OT is reduced, these neurons produced responses to frozen broadband noise that were more patterned at their preferred direction. This relationship held in the pooled data (Spearman correlation: azimuth: $r_{\mathrm{s}}=-0.58$; elevation: $r_{\mathrm{s}}=-0.39$; both $p<0.0001$; two-tailed $t$ test; Fig. 9E,F).

The onset of OT responses was removed from the analysis by calculating reproducibility $50 \mathrm{~ms}$ after the start of the stimulus (see Materials and Methods). In addition, to ensure patterning was not driven by the first evoked spike for each neuron, we also repeated the reproducibility analysis with the removal of the first spike after the 50-ms onset window. The correlation between reproducibility
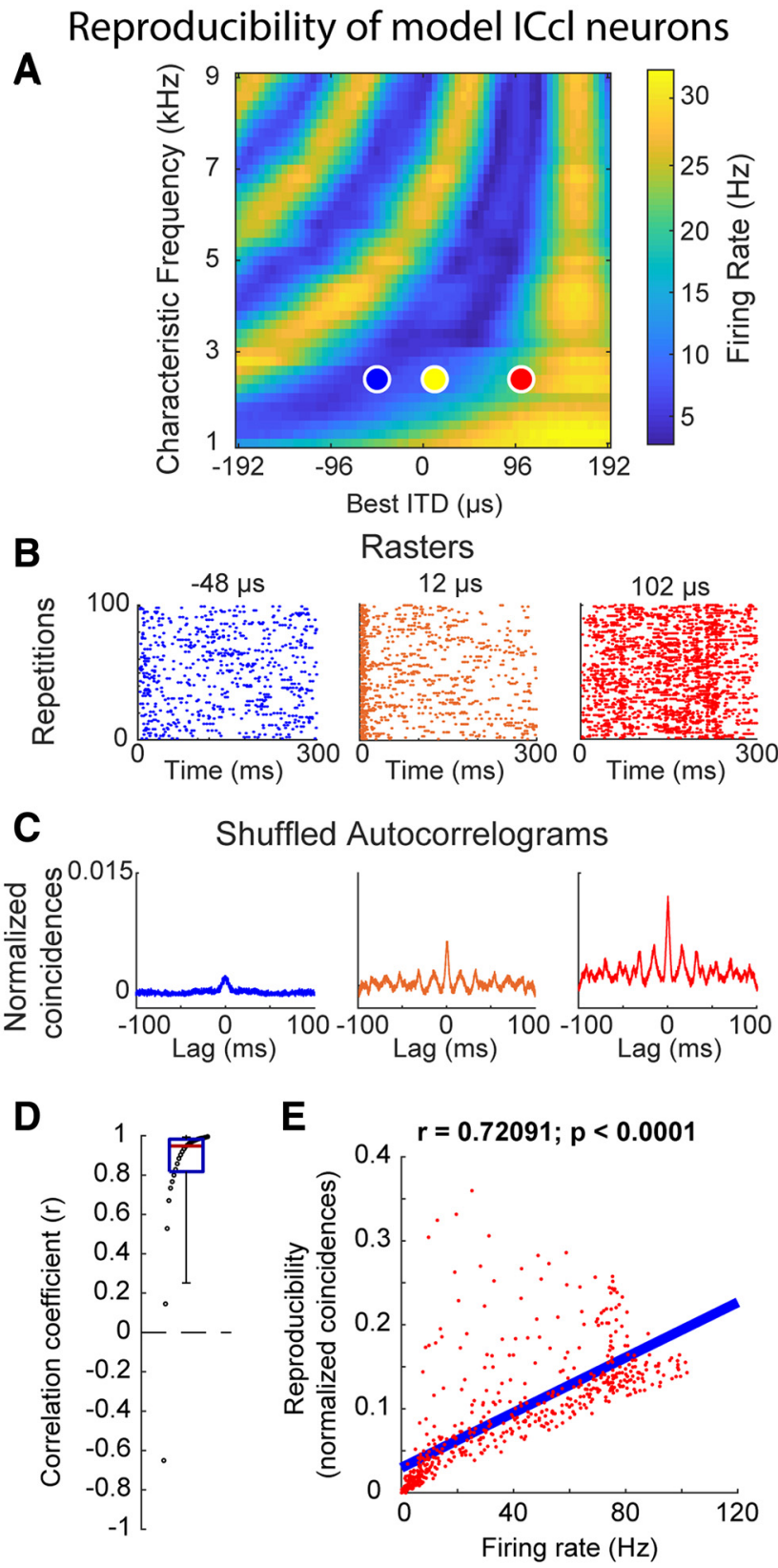

Figure 10. Relationship between reproducibility and firing rate in a modeled ICcl population. $\boldsymbol{A}$, Heat map of example population response of model ICcl neurons to a sound with $160-\mu \mathrm{s}$ ITD. Colored dots indicate the example neurons shown in $\boldsymbol{B}, \boldsymbol{C}$. $\boldsymbol{B}$, Example rasters of spikes for neurons shown in $\boldsymbol{A}$. $\boldsymbol{C}$, Example SACs for neurons shown in $\boldsymbol{A}$. $\boldsymbol{D}$, Correlation between reproducibility and firing rate for individual neurons in the population. Box plot shows the median correlation coefficient (red line), first and third quartile (blue box), 5th and 95th percentile (whiskers), and sorted data points. E, Relationship between firing rate and reproducibility for all data points pooled across model neurons. Blue line represents the linear regression. The correlation coefficient $(r)$ and exact $p$ value are above the plot. Data points in both panels are downsampled (one every 100; 560 out of 55,965 points plotted) to facilitate visualization, while statistical tests and linear regressions were calculated using all values. 
and firing rate remained significant for azimuth and elevation both for individual pairs (azimuth: $r=0.19 \pm 0.64$, $p=0.03$; elevation: $r=0.28 \pm 0.67, p=0.007$; two-tailed Wilcoxon signed-rank run on Z-scores) and across the sampled population (azimuth: $r=0.27$; elevation: $r=0.23$; both $p<0.0001$; two-tailed $t$ test). This analysis further validated that results are not significantly affected by the timing of first spikes in OT neurons' responses, which are characteristically locked to the onset of sound. We note that this further analysis could not be conducted for spike train distance because additional removal of spikes resulted in a substantial increase in the number of trials with absence of spikes, an exclusion criterion for this metric (see Materials and Methods).

Taken together, these results indicate that deep-layer OT neurons display synchronization with the envelope of frozen white noise stimuli at a reduced level compared with ICcl neurons, yet this pattern also varies in a stimulus-dependent manner.

\section{Stimulus-dependent synchrony in OT}

To address the questions whether temporal properties of population responses were stimulus-dependent in the map of space in a manner that could affect coding, spike synchrony of nearby OT neurons was examined. We hypothesized that the stimulus dependent temporal patterns underlying reproducible spiking in $\mathrm{ICcl}$ would lead to synchronized responses in similarly tuned ICcl neurons. We further hypothesized that synchronized inputs from ICcl neurons would more efficiently drive spiking in downstream space-map cells, having an impact on their temporal correlations.

These hypotheses were supported by a model of the sound localization pathway. We used a model of the auditory periphery (Fischer et al., 2009) to produce spiking responses of a population of ICcl neurons (Fig. 10A). The model included a cochlear filterbank that generated envelope fluctuations in different frequency channels for broadband noise stimuli. The cochlear filterbank outputs on the left and right were cross-correlated to model ITD detection. Gaussian noise was added to the cross-correlation output to model the input to ICcl neurons (Materials and Methods). Model ICcl neurons showed stimulus-dependent reproducibility in the experimentally observed range, both for individual neurons $(r=0.85 \pm 0.26$; twotailed $t$ test; Fig. $10 B-D$ ) and when data were pooled together ( $r=0.72 ; p<0.0001$, two-tailed $t$ test; Fig. 10E). This relationship was expected by the model's construction, where input signal-to-noise ratio is rate dependent. Interestingly, however, the modeled data displayed asymmetric variability around the regression line of reproducibility as a function of response strength (wider distribution above the regression line), reminiscent of the neural data (Figs. 4, 7).

Spike-time synchrony in the modeled populations was quantified by the integral of the standard-CCG and a shifted-CCG, which accounts for synchrony resulting from similar spike temporal patterns across trials. By construction, the model neurons displayed patterned spiking and thus the shifted-CCG accounted for nearly all synchrony in the standard-CCG, meaning the correctedCCG (see Materials and Methods) is non-informative in this case.

Supporting the hypotheses, model ICcl neurons with similar preferred ITDs showed stronger spike synchrony than neurons with differing preferred ITDs, quantified by both standard-CCG $(p<0.001$; two-tailed Mann-Whitney $U$; Fig. 11A, left) and shifted-CCG $(p<0.001$; two-tailed Mann-Whitney $U$; Fig. 11A, right). This is further demonstrated by the significant correlation between synchrony and GM firing rate observed for the standard-CCG $(r=0.94$; two-tailed $t$ test; $p<0.001)$ and shifted-CCG $(r=0.94 ; p<0.001$; two-tailed $t$ test; Fig. 11B).

The modeled ICCl neurons formed the input to a model of the midbrain auditory space map. In this map, best frequency is highly correlated with best ITD (Knudsen, 1984; Cazettes et al., 2014). By including this feature into the model, neurons with similar tunings fired synchronously. Figure $11 \mathrm{C}$ displays a comparison of synchrony assessed by standard-CCG (Fig. 11C, left) and shifted-CCG (Fig. $11 \mathrm{C}$, right) between the maximally responding unit in a population and neurons with similar ITD tuning $(<10-\mu \mathrm{s}$ preferred ITD) or very different ITD tunings $(>250-\mu \mathrm{s}$ preferred ITD). For both the standard-CCG and shiftedCCG, synchrony between nearby neurons was significantly stronger (both comparisons, $p<0.0001$; two-tailed Mann-Whitney $U$; Fig. 11C), matching the observation in the $\mathrm{ICcl}$ input population. Synchrony measured by both the standard-CCG $(r=0.995$; two-tailed $t$ test; $p<0.0001)$ and shifted-CCG ( $r=0.995$; two-tailed $t$ test; $p<0.0001)$ was positively correlated to the GM firing rate of the pair (Fig. 11D), further demonstrating the stimulus-dependent synchrony. This supports the hypothesis that stimulus dependence underlying reproducible spiking in $\mathrm{ICCl}$ would induce synchronized responses in similarly tuned neurons in $\mathrm{ICcl}$ and the space map.

The model's predictions were tested through in vivo recordings of nearby OT with tetrodes. Neurons were stimulated with repeated presentations of identical copies of noise delivered through speakers located at different positions (Materials and Methods). Synchrony and GM firing rate of simultaneously recorded pairs of neurons was quantified using the same methods as for modeled populations (Fig. 12A-C). To be included in the analysis, both neurons in a pair were required to display significant response to at least 1 stimulus location (2 SD above mean baseline). This criterion led to five pairs of neurons being omitted; however, all neurons had at least one usable pair ( $n=194$ original pairs, 189 usable pairs). Nearby neurons in OT fired synchronously, as previously reported (Beckert et al., 2017). As predicted by the model, synchrony (assessed by the standard-CCG) was correlated with the GM firing rate both across the sampled population $(r=0.31$; $p<0.0001$; two-tailed $t$ test; Fig. 12D) and in individual pairs of neurons $(r=0.31 \pm 0.25, W=16172 ; p<0.0001$; two-tailed Wilcoxon signed-rank run on Z-scores; Fig. 12E). We further examined whether stimulus dependent spiking patterns in OT neurons contributed to synchrony by computing a shifted-CCG (Materials and Methods). As observed for the standard-CCG, synchrony quantified by 


\section{Synchrony of model ICCl neurons}

A

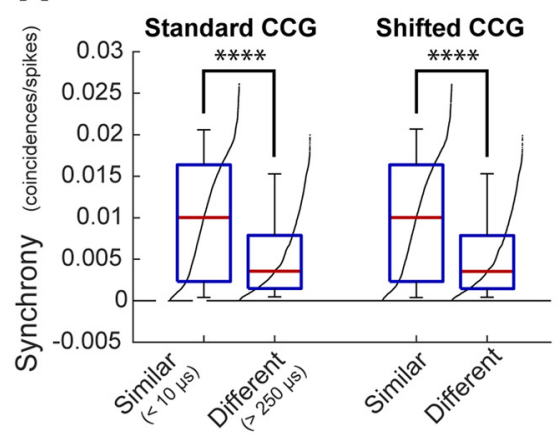

B

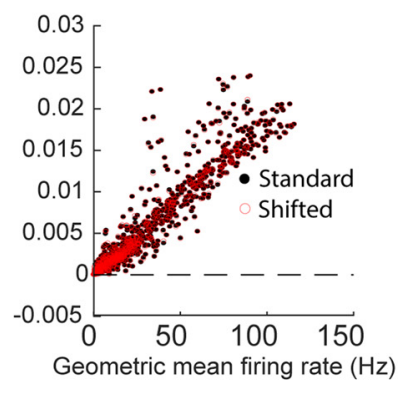

\section{Synchrony of model space-map neurons}

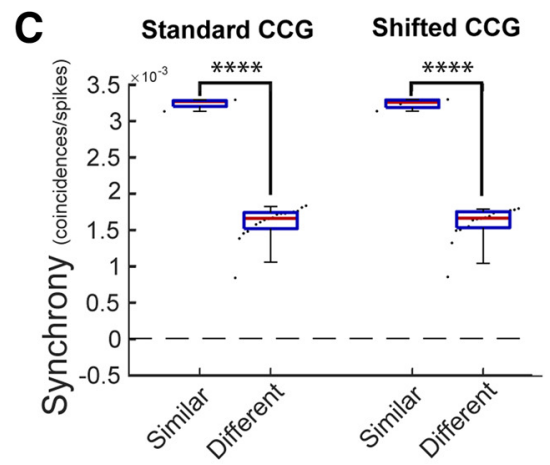

D

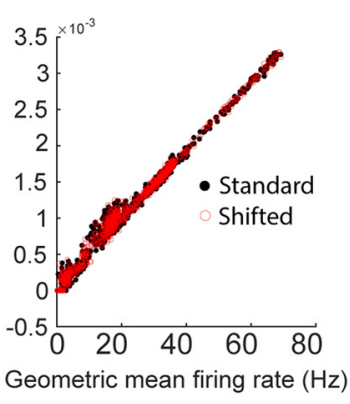

Figure 11. Stimulus-dependent synchrony in the modeled auditory midbrain. $\boldsymbol{A}$, Comparison of synchrony between neurons of the modeled ICcl population with the same preferred frequency and similar preferred ITD (difference in preferred ITD $<10 \mu$ s, "similar") and neurons with the same preferred frequency but different preferred ITDs (>250 $\mu$ s, "different"), measured by a standard-CCG (left) and a shifted-CCG (right). Definitions for similar and different ITD, specified in the $x$-axis labels for the standard-CCG group in $\boldsymbol{A}$, are used throughout the figure. $\boldsymbol{B}$, Relationship between synchrony and response strength (GM firing rate of pairs of neurons) for standard-CCG (black dots) and shifted-CCG (red circles). $\boldsymbol{C}$, Comparison of synchrony between neurons of the model space-map population tuned to similar ITDs at their peak response ( $\pm 10 \mu$ s from the maximum population response, similar) or between neurons with different preferred ITDs (difference $>250 \mu \mathrm{s}$, different), measured by a standard-CCG (left) and a shiftedCCG (right). $\boldsymbol{D}$, Same as $\boldsymbol{B}$ but for modeled space-map neurons. Box plots show the median synchrony (red line), first and third quartile (blue box), 5th and 95th percentile (whiskers), and raw data points. Data points in all plots are downsampled (one every $100)$ to facilitate visualization, while statistical tests, linear regression, and histogram were calculated using all values. MannWhitney $U$ test; ****p $<0.0001$.

the shifted-CCG was related to the firing rate of the neurons, both for individual pairs $(r=0.43 \pm 0.24, W=15061$; $p<0.0001$; two-tailed Wilcoxon signed-rank run on Zscores; Fig. 12E) and across the population $(r=0.63$; $p<0.0001$; two-tailed $t$ test; Fig. 12F). Synchrony quantified by the corrected-CCG, which removes coincidences due to shared patterning (see Materials and Methods), also displayed stimulus-dependency, but at a much weaker level than the standard and shifted-CCGs for individual pairs $(r=0.06 \pm 0.23, \quad W=7053 ; p=0.01$; two-tailed Wilcoxon signed-rank run on Z-scores; Fig. 12E). The correlation between firing rate and synchrony across the population was negative and weak ( $r=-0.036 ; p=0.0014$; two-tailed $t$ test; Fig. 12G). Together, this further suggests patterning is a fundamental determinant of synchrony.

As for the reproducibility analysis, spike synchrony was also computed excluding the first spike occurring after the removed 50-ms onset window. The correlation between synchrony measured by the standard-CCG and GM firing rate remained significant both for individual pairs $(r=0.25 \pm 0.35, p<0.0001$; two-tailed Wilcoxon signed-rank run on Z-scores) and across the sampled population ( $r=0.16, p<0.0001$; two-tailed $t$ test), as well as for the shifted-CCG for individual pairs $(r=0.31 \pm 0.29$, $p<0.0001$; two-tailed Wilcoxon signed-rank run on Zscores) and across the population ( $r=0.46, p<0.0001$; two-tailed $t$ test). Finally, as observed in the analysis including the first spike, the synchrony not related to patterning, measured by the corrected-CCG, was still stimulus dependent, yet at a weaker level, for individual pairs $(r=0.08 \pm 0.34, p=0.03$; two-tailed Wilcoxon signed-rank run on Z-scores), and negative and weak across the sampled population ( $r=-0.031, p=0.04$; two-tailed $t$ test). This consistency in the relationship after the removal of the first spike provides additional validation that the correlation 


\section{Synchrony in OT to varying sound direction}

A

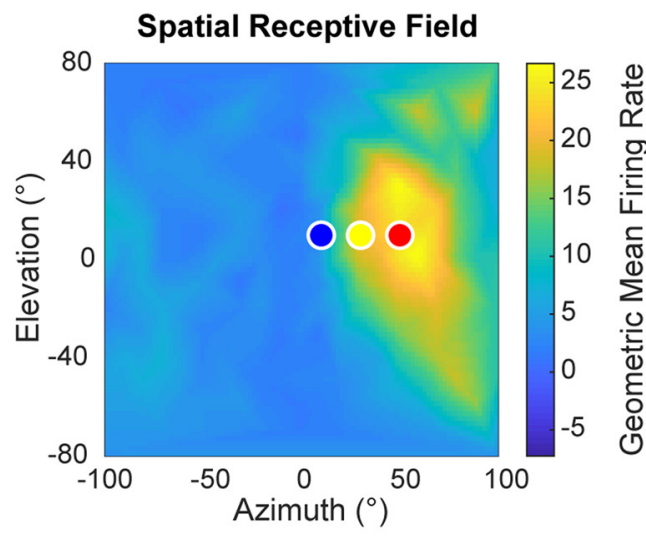

B

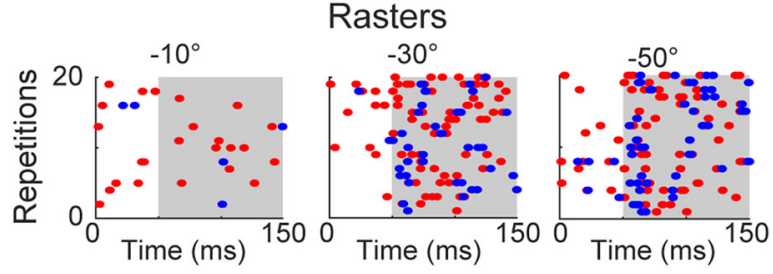

C

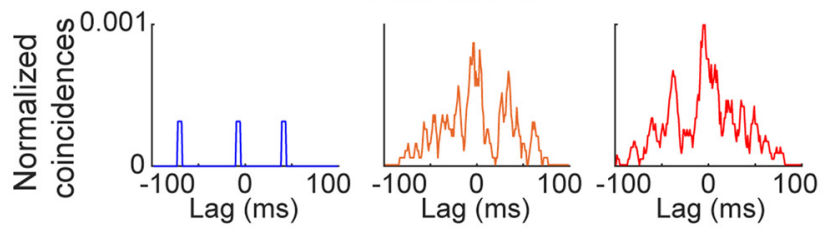

D
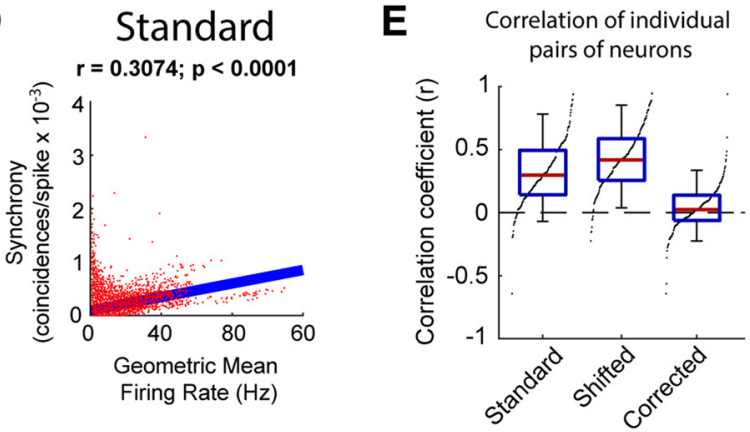

$\mathbf{F}$

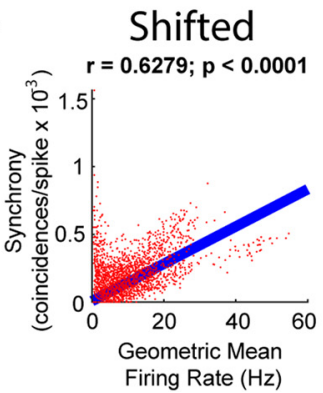

E

Standard CCG

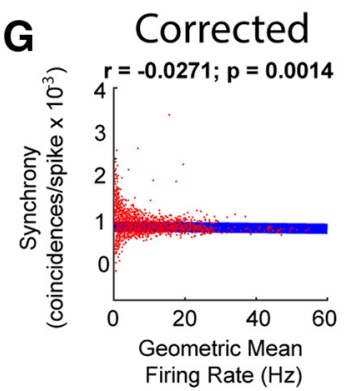

Figure 12. Relationship between synchrony and firing rate in OT, varying location in auditory space. $\boldsymbol{A}$, Example SpRF computed from the GM firing rate of a pair of OT neurons. Colored dots indicate example locations shown in $\boldsymbol{B}, \boldsymbol{C}$. $\boldsymbol{B}$, Example rasters for both neurons (neuron 1 in red and neuron 2 in blue) for the positions in space indicated in $\boldsymbol{A}$ (analysis window indicated

continued

by gray background). $\boldsymbol{C}$, Example standard-CCGs for locations indicated in $\boldsymbol{A}$. $\boldsymbol{D}$, Correlation between synchrony (measured by a standard-CCG) and response strength (GM firing rate) for pooled population data across pairs of neurons. The solid blue line is the linear regression across the entire sample. The correlation coefficient $(r)$ and exact $p$ value are shown above the plot. $\boldsymbol{E}$, Distribution of correlation coefficients $(r)$ between synchrony assessed by three metrics (standard-CCG, left; shifted-CCG, middle; and corrected-CCG, right) and the GM firing rate for each individual pair of neurons. The red line is the median, the upper and lower bounds of the blue box are the first and third quartile, and whiskers are 5th and 95th percentiles, individual points are all data sorted. $\boldsymbol{F}$, Correlation between synchrony related to patterning (shifted-CCG) and response strength for pooled population data of all pairs of recorded OT neurons. Same format as in panel $\boldsymbol{D}$. $\boldsymbol{G}$, Relationship of the remaining synchrony after subtraction of the shifted-CCG (correctedCCG) and response strength. Same format as in panel $\boldsymbol{D}$.

between response strength and reproducibility is not an effect of onset responses.

A possible source of synchrony in in vivo recordings of anesthetized preparations may be up-and-down states induced by anesthesia (Hahn et al., 2007; Ecker et al., 2014; Poskanzer and Yuste, 2016), where synchronized shifts in firing rate increase correlations (Mochol et al., 2015). To test for this effect, spontaneous activity was recorded in each site and a standard analysis for detecting these states (Pachitariu et al., 2015) was conducted (described in Materials and Methods). Only three out of 33 recordings met the criterion for the presence of up-states using this method $(0.7 \pm 2.8 \%$ of time in up-state). Thus, up-and-down states induced by anesthesia are an unlikely source of synchronization in this dataset. Additionally, midbrain neurons are typically more robust to changes due to anesthesia compared with forebrain neurons (Capsius and Leppelsack, 1996; Gaese and Ostwald, 2001; Syka et al., 2005; Schumacher et al., 2011; Karino et al., 2016). As such, we do not anticipate recordings in awake animals would differ significantly from the present results.

Taken together, synchrony of nearby OT neurons varied in a stimulus-dependent manner, and a substantial portion of this dependency could be explained by changes in the reproducible patterning of responses across trials. We then explored the potential effect of this phenomenon on the readout of the OT neural population using a model population of space-map neurons, presented in the next section below.

\section{Effect of stimulus-dependent synchrony on the readout of the space-map}

To test the effect of stimulus-dependent synchrony on the coding of sound location we modeled space-map neuron populations (Fig. 13A) where synchrony in the main and side peaks could either be stimulus dependent or equalized (example population response to a sound of 160- $\mu$ s ITD shown in Fig. 13A). To equalize synchrony in the main peaks and side peaks, we manipulated synchrony in responses to ITDs within the side peaks range by shifting 
the timing of spikes of the modeled population. As expected, this manipulation increased the synchrony in side peaks relative to the initial population, measured by the standard-CCG (initial $=0.0011 \pm 0.00003$, manipulated $=$ $0.0014 \pm 0.00003$ coincidences/spike; $p<0.0001$, twotailed $t$ test) as well as the shifted-CCG (initial $=$ $0.0011 \pm 0.00003$, manipulated $=0.0013 \pm 0.00003$ coincidences/spike; $p<0.0001$, two-tailed $t$ test).

To investigate the effect of spiking synchrony in the space map on the coding of ITD, we modeled the readout of the auditory space map by a downstream population of neurons for both the initial and manipulated populations (Materials and Methods). Consistent with our hypothesis, side peak heights of the space-map population readout were significantly larger when spike times were manipulated to enhance synchrony in side peaks (Fig. 13B,C; initial $=0.001 \pm 0.011 \%$; manipulated $=42 \pm 13 \%$ main peak; $p<0.0001$, two-tailed paired $t$ test).

Overall, this model shows that side peaks in the downstream output population were larger when synchrony was uniformly high across the population than when synchrony was stimulus dependent, as observed in OT. This suggests that stimulus-dependent synchrony may improve spatial discriminability in the readout of the OT map of space. Taken together, these findings demonstrate a transformation of auditory processing from the central nucleus of the inferior colliculus to the map-of-space where locking to the envelope is degraded while stimulus-dependent co-variability of spiking is preserved in a manner which may have an effect on the coding of sound location.

\section{Discussion}

This study reports stimulus-dependent across-trial reproducibility in the owl's midbrain nucleus ICcl and the downstream space-map in OT. Although OT neurons displayed lower precision compared with ICcl, synchrony of nearby OT cells was stimulus dependent, consistent with stimulusdependent reproducibility in ICcl. We further showed that this effect may improve coding of sound location.

\section{Stimulus-dependent reproducibility in ICcI}

Reproducibility in $\mathrm{ICcl}$ changed with firing rate as a function of binaural cues and sound direction. Similar stimulus-dependency of reproducibility has been described in mouse retinal ganglion cells (Murphy and Rieke, 2006), cat lateral geniculate nucleus (Cecchi et al., 2000), and rat stellate cells of the cerebellum (Suter and Jaeger, 2004). Thus, this relationship appears to generalize across multiple brain regions. However, we note that while this relationship may be anticipated for many sensory neurons, it is by no means obligatory in auditory neurons. For example, one of the input regions to ICcl, the posterior part of the dorsal lateral lemniscus (LLDp) displays maximal reproducibility when inhibitory input is strongest, not when firing rate is maximal, evidence that temporally precise inhibition can contribute to the reliability of envelope coding (Steinberg et al., 2013). Because the exact mechanism explaining how ILD tuning in ICcl emerges from inhibitory inputs originated in LLDp is still unresolved (Adolphs, 1993), the relationship between the
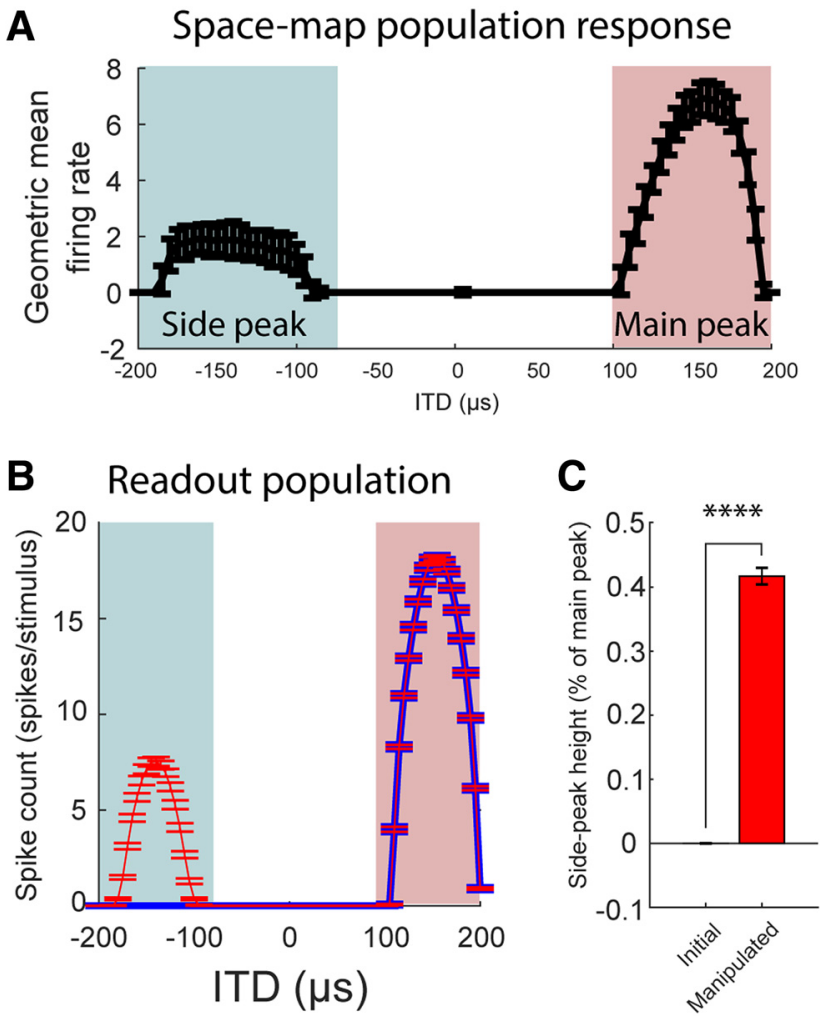

Figure 13. Synchrony dependent readout of a modeled spacemap population. A, Average population response of modeled space-map neurons to a sound at $160-\mu$ s ITD. Main and side peaks are highlighted in red and blue, respectively. $\boldsymbol{B}$, Average readout of the space-map from the initial (blue line) and manipulated (red line) modeled populations. Solid lines represent the mean and error bars are the SD. $\boldsymbol{C}$, Side peak heights relative to the main peak for initial and manipulated modeled populations across all ITDs tested. Paired $t$ test, $* * * * p<0.0001$.

ILD evoking maximal firing rate and reproducibility in ICcI cells and ILD tuning of their inputs from LLDp remains an intriguing question.

Specific mechanisms for stimulus-dependent reproducibility lie with the functional relationship between processes driving firing rate and spectrotemporal tuning of auditory neurons. It has been shown that sharper spectrotemporal tuning increases across-trial reproducibility by enhancing the selectivity of neurons to power fluctuations in the stimulus spectrum (Butts et al., 2007). Previous work has also demonstrated that spectrotemporal tuning of $\mathrm{ICCl}$ neurons sharpens with sound level (Keller and Takahashi, 2000). In addition, the computations of ILD and ITD in the brainstem pathways converging on ICCl (Wagner et al., 1987; Takahashi et al., 1989; Adolphs, 1993) are functionally linked to spectrotemporal tuning. As mentioned in the previous section above, in LLDp, where ILD is detected, inhibition modulates spectrotemporal tuning (Steinberg et al., 2013). In parallel, the ITDdetection mechanism in the nucleus laminaris (Carr and Konishi, 1990) is sensitive to time-dependent spectral features of sound (Fischer et al., 2011). Thus, the synchronization of spikes with spectrotemporal features of sounds is expected to change with stimulus parameters (sound 
level, ITD, and ILD) that also drive the firing rate of ICCl neurons. Our results are consistent with these predictions.

Frozen noise tokens used to assess temporal patterning when manipulating ITD and ILD were randomly varied to avoid potential biases caused by using single signals. The impact of using identical or randomly varying noise tokens is likely small because different examples of broadband white-noise stimuli would not contain large spectrotemporal differences. An interesting test, however, would be to compare reproducibility using the same tokens of naturalistic sounds as ITD and ILD are varied. Further work on this question could link the coding of sound identity and location, building on these initial observations.

Lastly, across stimulus conditions and midbrain areas, the correlation between response strength and the distance metric was stronger than between response strength and reproducibility. A reason for this difference may lie in the formulas used to compute each metric. The distance cost, by definition, cannot be larger than the number of spikes compared, which may result in lower variability when computed for weaker responses. The reproducibility metric, on the other hand, is normalized by firing rate, thus these values will always be between 0 and 1 regardless of number of spikes.

\section{Dichotic versus free-field stimulation}

In this study, we used two complementary stimulation protocols: headphones (dichotic) and free-field stimulation. Stimulus-dependency was observed for both forms of stimulation. Dichotic stimulation permits precise control of the sound signal reaching the eardrum, bypassing confounding factors present in the more naturalistic freefield stimulation, such as the filtering effect of the head. Free-field stimulation is subject to the filtering properties of the head, i.e., the HRTF, which induces co-variability of ITD and ILD (Keller et al., 1998) and variability of binaural cues across frequency and direction (Cazettes et al., 2014, 2016; Fischer and Peña, 2017). Thus, while observing this phenomenon for dichotic stimulation rules out changes in spectrotemporal properties of the sound induced by direction-dependent filtering of the owl's head and facial ruff as the sole cause of stimulus-dependent reproducibility, the observation that this dependency persisted for sounds in free-field indicates that the underlying mechanism is sufficient to modulate reproducibility in naturalistic conditions for sounds coming from different directions.

\section{Envelope coding in ICcl}

Synchronization with the sound envelope causes reproducible spike-time patterning in ICcl (Keller and Takahashi, 2000). Keller and Takahashi (2000) found no change in spike patterns in seven of eight neurons when sources were placed at different locations that produced similar spike counts, supporting the conclusion that spectrotemporal tuning of these neurons does not qualitatively change with position of the sound source. The present study does not contradict this finding, given it is not stating that the features neurons respond to differ, but only how reliably they respond to them across stimulus locations. Our observations further suggest that envelope coding by $\mathrm{ICcl}$ cells is likely dependent on their tuning to spatial cues. It has been hypothesized that locking to the envelope could improve the discrimination between concurrent sound sources at different locations (Keller and Takahashi, 2005) and between acoustic signals and their echoes (Baxter et al., 2013). Stimulus-dependent envelope coding would further strengthen this mechanism, as spiking dependence on the envelope will be reduced for sounds coming from a non-preferred location, making spike patterns of neurons preferring different directions more separable. In the current study, we investigated the effect of stimulus-dependent reproducibility in $\mathrm{ICcl}$ on population responses of the downstream OT map of auditory space. However, $\mathrm{ICcl}$ also sends projections to the forebrain through the primary auditory thalamus, nucleus ovoidalis (Proctor and Konishi, 1997; Arthur, 2005). The processing of sound may differ in the tectal and thalamic pathways to optimize the coding of sound location and high order spectral features, respectively, in a manner reminiscent of the proposed "where" and "what" pathways (Rauschecker and Tian, 2000; Kuwada et al., 2014). Studies in songbirds have shown a modest impact of sound source location on the coding of song identity by forebrain neurons, but a strong effect of sound location on competing sounds (Maddox et al., 2012). However, these studies were not performed on spatially tuned cells. Further studies can be performed to interrogate downstream effects of stimulus-dependent reproducibility in forebrain regions involved in sound localization.

\section{Stimulus-dependent reproducibility in OT}

Although OT neurons displayed reduced precision compared with $\mathrm{ICcl}$, as anticipated from previous studies of envelope and phase locking across brain regions in the auditory pathway (Johnson, 1980; Winter and Palmer, 1990; Köppl, 1997; Lu et al., 2001; Joris et al., 2004; Louage et al., 2004; Christianson and Peña, 2006, 2007; Liu et al., 2006; Steinberg and Peña, 2011; Yao et al., $2015 a, b)$, their reproducibility was stimulus dependent. This trend is consistent with the notion of stimulus-dependent synchrony in upstream neurons of ICcl driving reproducible firing in OT.

The weaker patterning of recorded (Fig. 8) and modeled (Fig. 10) OT cells relative to $\mathrm{ICcl}$ neurons may be due to the broad frequency tuning of space-map neurons that results in a reduction of envelope fluctuations driving OT neurons. Intracellular in vivo recordings of ICx cells show strong threshold adaptation (Pena and Konishi, 2002), resulting in fast depolarizations producing spikes with greater fidelity (Platkiewicz and Brette, 2011; Fontaine et al., 2014a,b). Sound stimulation by broadband noise bursts, as used in our study, results in a strong and fast depolarization at the beginning of the stimulus, while 
during the sustained portion of the stimulus neurons are tonically depolarized, resulting in slower depolarization rates after the onset (Pena and Konishi, 2002). Previous modeling work has demonstrated that slower depolarizations are more vulnerable to noise in the membrane potential (Mainen and Sejnowski, 1995; Berry et al., 1997; Cecchi et al., 2000). Thus, it is expected that spikes occurring after onset responses show less reproducible patterns. OT neurons may also lock to the envelope of stimuli featuring strong correlated modulations, as in sinusoidalamplitude modulated signals (Keller and Takahashi, 2000, 2005; Nelson and Takahashi, 2010) or complex natural sounds (Escabi and Schreiner, 2002).

\section{Stimulus-dependent synchrony in OT}

Synchrony in OT was stimulus dependent. Our study indicates that a cause of the stimulus-dependent synchrony in OT may be the stimulus-dependent spiking patterns observed in OT and the upstream ICcl. Cochlear filters act as bandpass filters for broadband sounds (Evans, 1989). Because ICcl neurons display narrow frequency tuning, the envelope of their input is expected to strongly fluctuate even for white noise stimuli (Smith, 1997, 2011). Assuming the across-trial reproducibility is a proxy for the precision of spike timing within a given trial, ICcl neurons with the same frequency tuning will undergo strong likelihood of firing more simultaneously as their synchronization with the envelope increases.

The frequency convergence from ICcl into ICx causes these neurons to display frequency tuning within a few kilohertz range (Knudsen and Konishi, 1978a; Knudsen, 1984; Mazer, 1998; Singheiser et al., 2012). Although the broader frequency tuning may attenuate envelope fluctuations under white noise stimulation, ICx neurons remain sensitive to transient depolarizations (Fontaine et al., 2014b). Small amounts of synchronous input among noisy background activity is sufficient to greatly impact spiking efficiency (Rossant et al., 2011). Thus, ICx neurons with similar frequency tuning may display synchronous spiking due to ICcl neurons' envelope dependent spiking patterns. Mechanistically, this synchrony does not require shared inputs, as the correlations driving the activity exist within the stimulus itself.

At non-preferred sound directions, ICcl neurons will track the sound's envelope with lower fidelity, resulting in reduced synchrony across frequency channels in ICcl, which will then be relayed downstream to ICx. We therefore hypothesize that binaural cue-dependent synchrony in ICcl underlies binaural cue-dependent synchrony downstream to ICx. Lastly, stimulus-dependent synchrony could be inherited from ICx to OT through the known shared point-topoint projections between these structures (Knudsen and Knudsen, 1983; Beckert et al., 2017). Simultaneous recording of nearby ICcl and ICx cells has proven challenging due to large evoked potentials corrupting spike sorting (Beckert MV and Pena JL unpublished observations). Thus, new techniques will be required to demonstrate that stimulusdependent synchrony is also observed upstream of OT.

\section{Effect of stimulus-dependent synchrony on coding}

While some studies have considered synchronous spiking detrimental to the coding capacity of a system by reducing temporal sparseness (Itskov and Abbott, 2008; Srivastava et al., 2008; Wiechert et al., 2010), an alternative interpretation is that synchronous firing may be beneficial by increasing the signal-to-noise ratio through temporal summation (Abeles, 1982; Alonso et al., 1996; Usrey et al., 1998; Moreno et al., 2002; Kenyon et al., 2004; Brette, 2012).

Through modeling, we show that side peaks in the population response of the modeled output population were either reduced or completely eliminated when synchrony is stimulus dependent. Assuming that stimulus-dependent synchrony is already present in ICcl, this modeling suggests a mechanism for disambiguating ITD, in addition to the proposed frequency convergence from ICcl to ICx (Takahashi and Konishi, 1986; Trahiotis and Stern, 1989; Mazer, 1998; Peña and Konishi, 2000) for resolving the ambiguity of phase information in the central auditory pathway. Additionally, our study shows that, even if synchrony in OT were a residual effect from its input, a local readout of nearby OT neurons could still enhance accuracy of representing auditory space in an output population. OT neurons are known to project to premotor nuclei that control the owl's head movement (Masino and Knudsen, 1992, 1993). While the only study of auditory responses of neurons in the motor-associated medial tegmental nucleus that controls the owl's head movement demonstrates a convergent projection from the midbrain map onto these premotor cells (Cazettes et al., 2018), this study also showed that removing side peaks was a critical aspect of reading out OT's population to produce observed premotor tegmentum responses, further supporting the functional relevance of stimulus-dependent synchrony in OT for coding auditory space.

\section{Conclusions}

We have demonstrated that the across-trial precision of spike timing of neurons in the barn owl midbrain is stimulus dependent, increasing at the neurons' preferred direction. Though this patterning dissipates in the downstream map-of-space, a stimulus-dependent synchrony is observed. Modeling shows that this stimulus dependent synchrony may have functional implications for the coding of auditory space, thus suggesting a functional interaction between the tuning to spectrotemporal features and to sound location in auditory neurons. Stimulus-dependent reproducibility and synchrony are consistent with multiple cellular and network mechanisms affecting spiking precision proposed in other brain regions (Mainen and Sejnowski, 1995; Berry et al., 1997; Berry and Meister, 1998; Cecchi et al., 2000; Butts et al., 2007), suggesting these findings are 
generalizable across neurons sensitive to the temporal structure of the stimulus.

\section{References}

Abeles M (1982) Role of the cortical neuron: integrator or coincidence detector? Isr J Med Sci 18:83-92.

Adolphs R (1993) Bilateral inhibition generates neuronal responses tuned to interaural level differences in the auditory brainstem of the barn owl. J Neurosci 13:3647-3668.

Alonso JM, Usrey WM, Reid RC (1996) Precisely correlated firing in cells of the lateral geniculate nucleus. Nature 383:815-819.

Aronov D, Reich DS, Mechler F, Victor JD (2003) Neural coding of spatial phase in V1 of the macaque monkey. J Neurophysiol 89:3304-3327.

Arthur BJ (2005) Distribution within the barn owl's inferior colliculus of neurons projecting to the optic tectum and thalamus. J Comp Neurol 492:110-121.

Atencio CA, Sharpee TO, Schreiner CE (2012) Receptive field dimensionality increases from the auditory midbrain to cortex. J Neurophysiol 107:2594-2603.

Bair W, Zohary E, Newsome WT (2001) Correlated firing in macaque visual area MT: time scales and relationship to behavior. J Neurosci 21:1676-1697.

Baxter CS, Nelson BS, Takahashi TT (2013) The role of envelope shape in the localization of multiple sound sources and echoes in the barn owl. J Neurophysiol 109:924-931.

Beckert MV, Pavão R, Peña JL (2017) Distinct correlation structure supporting a rate-code for sound localization in the owl's auditory forebrain. eNeuro 4:ENEURO.0144-17.2017.

Berry MJ, Meister M (1998) Refractoriness and neural precision. J Neurosci 18:2200-2211.

Berry MJ, Warland DK, Meister M (1997) The structure and precision of retinal spike trains. Proc Natl Acad Sci USA 94:5411-5416.

Bragin A, Benassi SK, Engel J (2012) Patterns of the UP-down state in normal and epileptic mice. Neuroscience 225:76-87.

Bremen P, Poganiatz I, von Campenhausen M, Wagner H (2007) Sensitivity to interaural time difference and representation of azimuth in central nucleus of inferior colliculus in the barn owl. J Comp Physiol A Neuroethol Sens Neural Behav Physiol 193:99112.

Brette R (2012) Computing with neural synchrony. PLoS Comput Biol 8:e1002561.

Butts DA, Weng C, Jin J, Yeh C-I, Lesica NA, Alonso J-M, Stanley GB (2007) Temporal precision in the neural code and the timescales of natural vision. Nature 449:92-95.

Calabrese A, Woolley SMN (2015) Coding principles of the canonical cortical microcircuit in the avian brain. Proc Natl Acad Sci USA 112:3517-3522.

Capsius B, Leppelsack HJ (1996) Influence of urethane anesthesia on neural processing in the auditory cortex analogue of a songbird. Hear Res 96:59-70.

Carr CE, Konishi M (1990) A circuit for detection of interaural time differences in the brain stem of the barn owl. J Neurosci 10:32273246.

Cazettes F, Fischer BJ, Pena JL (2014) Spatial cue reliability drives frequency tuning in the barn owl's midbrain. Elife 3:e04854.

Cazettes F, Fischer BJ, Peña JL (2016) Cue reliability represented in the shape of tuning curves in the owl's sound localization system. J Neurosci 36:2101-2110.

Cazettes F, Fischer BJ, Beckert MV, Pena JL (2018) Emergence of an adaptive command for orienting behavior in premotor brainstem neurons of barn owls. J Neurosci 38:7270-7279.

Cecchi GA, Sigman M, Alonso JM, Martínez L, Chialvo DR, Magnasco MO (2000) Noise in neurons is message dependent. Proc Natl Acad Sci USA 97:5557-5561.

Christianson GB, Peña JL (2006) Noise reduction of coincidence detector output by the inferior colliculus of the barn owl. J Neurosci 26:5948-5954.
Christianson GB, Peña JL (2007) Preservation of spectrotemporal tuning between the nucleus laminaris and the inferior colliculus of the barn owl. J Neurophysiol 97:3544-3553.

du Lac S, Knudsen El (1990) Neural maps of head movement vector and speed in the optic tectum of the barn owl. J Neurophysiol 63:131-146.

Ecker AS, Berens P, Cotton RJ, Subramaniyan M, Denfield GH, Cadwell CR, Smirnakis SM, Bethge M, Tolias AS (2014) State dependence of noise correlations in macaque primary visual cortex. Neuron 82:235-248.

Escabi MA, Schreiner CE (2002) Nonlinear spectrotemporal sound analysis by neurons in the auditory midbrain. J Neurosci 22:41144131.

Evans EF (1989) Cochlear filtering: a view seen through the temporal discharge patterns of single cochlear nerve fibres. In: Cochlear mechanisms: structure, function, and models (Wilson JP, Kemp DT, eds), pp 241-250. Boston: Springer US.

Fischer BJ, Peña JL (2017) Optimal nonlinear cue integration for sound localization. J Comput Neurosci 42:37-52.

Fischer BJ, Peña JL, Konishi M (2007) Emergence of multiplicative auditory responses in the midbrain of the barn owl. $\mathrm{J}$ Neurophysiol 98:1181-1193.

Fischer BJ, Anderson CH, Peña JL (2009) Multiplicative auditory spatial receptive fields created by a hierarchy of population codes. PLoS One 4:e8015.

Fischer BJ, Steinberg LJ, Fontaine B, Brette R, Peña JL (2011) Effect of instantaneous frequency glides on interaural time difference processing by auditory coincidence detectors. Proc Natl Acad Sci USA 108:18138-18143.

Fontaine B, MacLeod KM, Lubejko ST, Steinberg LJ, Köppl C, Peña $\mathrm{JL}$ (2014a) Emergence of band-pass filtering through adaptive spiking in the owl's cochlear nucleus. J Neurophysiol 112:430445.

Fontaine B, Peña JL, Brette R (2014b) Spike-threshold adaptation predicted by membrane potential dynamics in vivo. PLoS Comput Biol 10:e1003560.

Gaese BH, Ostwald J (2001) Anesthesia changes frequency tuning of neurons in the rat primary auditory cortex. J Neurophysiol 86:1062-1066.

Hahn TTG, Sakmann B, Mehta MR (2007) Differential responses of hippocampal subfields to cortical up-down states. Proc Natl Acad Sci USA 104:5169-5174.

Itskov V, Abbott LF (2008) Pattern capacity of a perceptron for sparse discrimination. Phys Rev Lett 101:018101.

Johnson DH (1980) The relationship between spike rate and synchrony in responses of auditory-nerve fibers to single tones. $J$ Acoust Soc Am 68:1115-1122.

Joris PX, Schreiner CE, Rees A (2004) Neural processing of amplitude-modulated sounds. Physiol Rev 84:541-577.

Joris PX, Louage DH, Cardoen L, van der Heijden M (2006) Correlation index: a new metric to quantify temporal coding. Hear Res 216-217:19-30.

Karino G, George I, Loison L, Heyraud C, De Groof G, Hausberger M, Cousillas H (2016) Anesthesia and brain sensory processing: impact on neuronal responses in a female songbird. Sci Rep 6:39143.

Keller CH, Takahashi TT (2000) Representation of temporal features of complex sounds by the discharge patterns of neurons in the owl's inferior colliculus. J Neurophysiol 84:2638-2650.

Keller CH, Takahashi TT (2005) Localization and identification of concurrent sounds in the owl's auditory space map. J Neurosci 25:10446-10461.

Keller CH, Hartung K, Takahashi TT (1998) Head-related transfer functions of the barn owl: measurement and neural responses. Hear Res 118:13-34.

Kenyon GT, Theiler J, George JS, Travis BJ, Marshak DW (2004) Correlated firing improves stimulus discrimination in a retinal model. Neural Comput 16:2261-2291. 
Kim G, Doupe A (2011) Organized representation of spectrotemporal features in songbird auditory forebrain. J Neurosci 31:1697716990.

Knudsen El (1982) Auditory and visual maps of space in the optic tectum of the owl. J Neurosci 2:1177-1194.

Knudsen El (1984) Auditory properties of space-tuned units in owl's optic tectum. J Neurophysiol 52:709-723.

Knudsen El, Konishi M (1978a) Space and frequency are represented separately in auditory midbrain of the owl. J Neurophysiol 41:870884.

Knudsen El, Konishi M (1978b) A neural map of auditory space in the owl. Science 200:795-797.

Knudsen El, Knudsen PF (1983) Space-mapped auditory projections from the inferior colliculus to the optic tectum in the barn owl (Tyto alba). J Comp Neurol 218:187-196.

Knudsen El, Blasdel GG, Konishi M (1979) Sound localization by the barn owl (Tyto alba) measured with the search coil technique. J Comp Physiol 133:1-11.

Kohn A, Smith MA (2005) Stimulus dependence of neuronal correlation in primary visual cortex of the macaque. J Neurosci 25:36613673.

Köppl C (1997) Phase locking to high frequencies in the auditory nerve and cochlear nucleus magnocellularis of the barn owl, Tyto alba. J Neurosci 17:3312-3321.

Köppl C, Carr CE (2003) Computational diversity in the cochlear nucleus angularis of the barn owl. J Neurophysiol 89:2313-2329.

Kuwada S, Bishop B, Kim DO (2014) Azimuth and envelope coding in the inferior colliculus of the unanesthetized rabbit: effect of reverberation and distance. J Neurophysiol 112:1340-1355.

Liu LF, Palmer AR, Wallace MN (2006) Phase-locked responses to pure tones in the inferior colliculus. J Neurophysiol 95:19261935.

Louage DHG, van der Heijden M, Joris PX (2004) Temporal properties of responses to broadband noise in the auditory nerve. $J$ Neurophysiol 91:2051-2065.

Lu T, Liang L, Wang X (2001) Temporal and rate representations of time-varying signals in the auditory cortex of awake primates. Nat Neurosci 4:1131-1138.

Machens CK, Prinz P, Stemmler MB, Ronacher B, Herz AVM (2001) Discrimination of behaviorally relevant signals by auditory receptor neurons. Neurocomputing 38-40:263-268.

Maddox RK, Billimoria CP, Perrone BP, Shinn-Cunningham BG, Sen $\mathrm{K}$ (2012) Competing sound sources reveal spatial effects in cortical processing. PLoS Biol 10:e1001319.

Mainen ZF, Sejnowski TJ (1995) Reliability of spike timing in neocortical neurons. Science 268:1503-1506.

Masino T, Knudsen El (1992) Anatomical pathways from the optic tectum to the spinal cord subserving orienting movements in the barn owl. Exp Brain Res 92:194-208.

Masino T, Knudsen El (1993) Orienting head movements resulting from electrical microstimulation of the brainstem tegmentum in the barn owl. J Neurosci 13:351-370.

Mazer JA (1998) How the owl resolves auditory coding ambiguity. Proc Natl Acad Sci USA 95:10932-10937.

McFarland JM, Hahn TTG, Mehta MR (2011) Explicit-duration hidden Markov model inference of UP-DOWN states from continuous signals. PLoS One 6:e21606.

Middlebrooks JC, Clock AE, Xu L, Green DM (1994) A panoramic code for sound location by cortical neurons. Science 264:842844.

Mochol G, Hermoso-Mendizabal A, Sakata S, Harris KD, de la Rocha J (2015) Stochastic transitions into silence cause noise correlations in cortical circuits. Proc Natl Acad Sci USA 112:3529-3534.

Moiseff A (1989) Bi-coordinate sound localization by the barn owl. J Comp Physiol A Neuroethol Sens Neural Behav Physiol 164:637644.

Moreno R, de la Rocha J, Renart A, Parga N (2002) Response of spiking neurons to correlated inputs. Phys Rev Lett 89:288101.
Murphy GJ, Rieke F (2006) Network variability limits stimulus-evoked spike timing precision in retinal ganglion cells. Neuron 52:511524.

Nelson BS, Takahashi TT (2010) Spatial hearing in echoic environments: the role of the envelope in owls. Neuron 67:643-655.

Pachitariu M, Lyamzin DR, Sahani M, Lesica NA (2015) State-dependent population coding in primary auditory cortex. J Neurosci 35:2058-2073.

Parga N, Abbott LF (2007) Network model of spontaneous activity exhibiting synchronous transitions between up and down States. Front Neurosci 1:57-66.

Pena JL, Konishi M (2002) From postsynaptic potentials to spikes in the genesis of auditory spatial receptive fields. J Neurosci 22:5652-5658.

Peña JL, Konishi M (2000) Cellular mechanisms for resolving phase ambiguity in the owl's inferior colliculus. Proc Natl Acad Sci USA 97:11787-11792.

Platkiewicz J, Brette R (2011) Impact of fast sodium channel inactivation on spike threshold dynamics and synaptic integration. PLoS Comput Biol 7:e1001129.

Poskanzer KE, Yuste R (2016) Astrocytes regulate cortical state switching in vivo. Proc Natl Acad Sci USA 113:E2675-E2684.

Proctor L, Konishi M (1997) Representation of sound localization cues in the auditory thalamus of the barn owl. Proc Natl Acad Sci USA 94:10421-10425.

Rauschecker JP, Tian B (2000) Mechanisms and streams for processing of "what" and "where" in auditory cortex. Proc Natl Acad Sci USA 97:11800-11806.

Rossant C, Leijon S, Magnusson AK, Brette R (2011) Sensitivity of noisy neurons to coincident inputs. J Neurosci 31:17193-17206.

Schumacher JW, Schneider DM, Woolley SMN (2011) Anesthetic state modulates excitability but not spectral tuning or neural discrimination in single auditory midbrain neurons. J Neurophysiol 106:500-514

Singheiser M, Gutfreund Y, Wagner H (2012) The representation of sound localization cues in the barn owl's inferior colliculus. Front Neural Circuits 6:45.

Smith JO III (2011) Spectral audio signal processing. Stanford, CA: W3k Publishing.

Smith MA, Kohn A (2008) Spatial and temporal scales of neuronal correlation in primary visual cortex. J Neurosci 28:1259112603.

Smith SW (1997) The scientist and engineer's guide to digital signal processing, Ed 1. Poway: California Technical Publishing.

Srivastava V, Parker DJ, Edwards SF (2008) The nervous system might "orthogonalize" to discriminate. J Theor Biol 253:514517.

Steinberg LJ, Peña JL (2011) Difference in response reliability predicted by spectrotemporal tuning in the cochlear nuclei of barn owls. J Neurosci 31:3234-3242.

Steinberg LJ, Fischer BJ, Peña JL (2013) Binaural gain modulation of spectrotemporal tuning in the interaural level difference-coding pathway. J Neurosci 33:11089-11099.

Suter KJ, Jaeger D (2004) Reliable control of spike rate and spike timing by rapid input transients in cerebellar stellate cells. Neuroscience 124:305-317.

Syka J, Suta D, Popelár J (2005) Responses to species-specific vocalizations in the auditory cortex of awake and anesthetized guinea pigs. Hear Res 206:177-184.

Takahashi T, Konishi M (1986) Selectivity for interaural time difference in the owl's midbrain. J Neurosci 6:3413-3422.

Takahashi T, Moiseff A, Konishi M (1984) Time and intensity cues are processed independently in the auditory system of the owl. J Neurosci 4:1781-1786.

Takahashi TT, Wagner H, Konishi M (1989) Role of commissural projections in the representation of bilateral auditory space in the barn owl's inferior colliculus. J Comp Neurol 281:545-554.

Trahiotis C, Stern RM (1989) Lateralization of bands of noise: effects of bandwidth and differences of interaural time and phase. $J$ Acoust Soc Am 86:1285-1293. 
Usrey WM, Reppas JB, Reid RC (1998) Paired-spike interactions and synaptic efficacy of retinal inputs to the thalamus. Nature 395:384-387.

Victor JD (2005) Spike train metrics. Curr Opin Neurobiol 15:585592.

Victor JD, Purpura KP (1996) Nature and precision of temporal coding in visual cortex: a metric-space analysis. J Neurophysiol 76:1310-1326.

Wagner H, Takahashi T, Konishi M (1987) Representation of interaural time difference in the central nucleus of the barn owl's inferior colliculus. J Neurosci 7:3105-3116.

Wiechert MT, Judkewitz B, Riecke H, Friedrich RW (2010) Mechanisms of pattern decorrelation by recurrent neuronal circuits. Nat Neurosci 13:1003-1010.
Winter IM, Palmer AR (1990) Responses of single units in the anteroventral cochlear nucleus of the guinea pig. Hear Res 44:161-178.

Woolley SMN, Gill PR, Fremouw T, Theunissen FE (2009) Functional groups in the avian auditory system. J Neurosci 29:2780-2793.

Xu X, Wang R (2014) Neurodynamics of up and down transitions in a single neuron. Cogn Neurodyn 8:509-515.

Yao JD, Bremen P, Middlebrooks JC (2015a) Emergence of spatial stream segregation in the ascending auditory pathway. J Neurosci 35:16199-16212.

Yao JD, Bremen P, Middlebrooks JC (2015b) Transformation of spatial sensitivity along the ascending auditory pathway. J Neurophysiol 113:3098-3111. 